\title{
Sample Path Large Deviations for Diffusion Processes on Configuration Spaces over a Riemannian Manifold
}

By

Michael RöcKnER* and Tusheng S. ZHANG**

\begin{abstract}
In this paper, we establish a sample path large deviation principle for a class of diffusion processes on configuration spaces over a Riemannian manifold. The rate functional turns out to be the energy of the paths associated to the $L^{2}$-Wasserstein distance.
\end{abstract}

\section{Contents}

$\S 1 . \quad$ Introduction

$\S 2$. Framework

$\S 3 . \quad$ Some Preliminary Exponential Estimates

$\S 4$. Large Deviation Estimates for Finite Dimensional Projections: Upper Bounds

$\S 5$. Identification of the Rate Functional

$\S 6$. Large Deviation Estimates for Finite Dimensional Projections: the Lower Bounds

Communicated by Y. Takahashi. Received January 7, 2003.

2000 Mathematics Subject Classification(s): 60G60, 60J60, 60G57, 60G55, 31C25

Key words and phrases: Dirichlet forms, intrinsic metric, large deviations, configuration spaces, energy of path, Girsanov transform.

*Fakultät für Mathematik, Universität Bielefeld, Universitätsstr. 25, D-33615 Bielefeld, Germany.

e-mail: roeckner@mathematik.uni-bielefeld.de

** Department of Mathematics, University of Manchester, Oxford Road, Manchester M13 9PL, England, U.K.

e-mail: tzhang@ma.man.ac.uk 
§7. The Sample Path Large Deviations

$\S 8$. Interacting Case

Acknowledgement

References

\section{$\S 1$. Introduction}

Since Varadhan's work [Var67] on large deviations for the small time asymptotics for diffusion processes a large number of papers has been devoted to this subject. For diffusions on finite dimensional state spaces we would like to mention here particularly Norris's work [Nor97] and also the references therein. In recent years, small time large deviations of diffusions have also been studied in infinite dimensions, (see [Fan94, FZ99, AZ02, Hin02, Ram01, Sch96, Zha00]).

In this paper we prove a small time large deviation principle for a class of diffusions on configuration space (i.e., infinite particle systems in continuum) on the sample path level. The paper in the literature, which is closest to our situation, is the paper by Schied for the case of the super-Brownian motion. Our diffusions, however, take values in $\Gamma_{M}$, i.e., the space of all $\mathbf{Z}_{+} \cup\{+\infty\}$ valued Radon measures on a finite dimensional, connected complete Riemannian manifold $M$. So, (at least if $M$ is not compact) the diffusions on $\Gamma_{M}$ can be heuristically written as

$$
X_{t}=\sum_{i=1}^{\infty} \delta_{X_{t}^{i}},
$$

representing interacting random particles. One way to construct such a diffusion is to use the theory of Dirichlet forms (see, for example, [MR00, AKR96a]). The geometry and analysis on configuration space $\Gamma_{M}$ was carried out in [AKR96a, AKR96b, AKR98a, AKR98b]. The intrinsic metric of the associated Dirichlet form was identified as an $L^{2}$-Wasserstein type distance in [RS99]. As said before in this paper, we establish a sample path large deviation principle for the diffusion process $X_{t}, t \geq 0$, on the path space $\Omega:=C\left([0,1] \rightarrow \Gamma_{M}\right)$. Our strategy is to first establish the principle for the so called Brownian motion on configuration space i.e., the independent particle process on $M$, which is the harder part, and then to obtain the large deviation principle for more general diffusions via Girsanov transformation. The rate functional turns out to be the energy of the paths associated to the $L^{2}$-Wasserstein type distance from [RS99]. When the manifold $M$ is the real line, the small time asymptotics (not the sample path large deviation) was analyzed in [Zha01]. 
We want to emphasize that in the case of the independent particle process we do not use its construction by Dirichlet forms, but rather the pathwise construction from [KLR03], which (as shown in [KLR03]) is possible for an explicitly described set $\Gamma_{\infty} \subset \Gamma_{M}$ of initial configurations. $\Gamma_{\infty}$ is also an invariant set for the process, i.e., $\left(X_{t}\right)_{t \geq 0}$ stays in $\Gamma_{\infty}$ for all times. We, therefore, can prove the sample path large deviation principle for all initial conditions $\gamma \in \Gamma_{\infty}$. We also use the metric $d_{\infty}$ introduced on $\Gamma_{\infty}$ in [KLR03] in a decisive way. $d_{\infty}$ induces a stronger topology on $\Gamma_{\infty}$ than the vague topology and is crucially used in Section 6 below (cf. Theorem 6.8).

The paper is organized as follows. In Section 2 we present our framework giving all conditions on $M$ used below. We also recall relevant definitions and results from [KLR03]. In Section 3, we prove exponential estimates which are necessary for the sequel. Section 4 is devoted to the upper bound estimates for finite dimensional projections of the diffusion. In Section 5 the rate functional is identified. The lower bound estimates for finite dimensional projections are discussed in Section 6. The sample path large deviation principle is finally proved in Section 7. In Section 8, we establish the large deviation principle for a more general class of diffusions.

\section{§2. Framework}

Let $M$ be a complete, connected Riemannian manifold as in the introduction. For simplicity we assume that $M$ has dimension bigger than two. Let $p_{t}(x, y)$ denote the heat kernel on $M$. Throughout the paper, we assume that the manifold $M$ satisfies the following conditions:

A.1. For any $\delta>0$ there exists a constant $c_{1}(\delta)$ such that

$$
\frac{\exp \left[-\frac{d(x, y)^{2}}{(2-\delta) t}\right]}{c_{1}(\delta) m(B(y, \sqrt{t}))} \leq p_{t}(x, y) \leq c_{1}(\delta) \frac{\exp \left[-\frac{d(x, y)^{2}}{(2+\delta) t}\right]}{m(B(y, \sqrt{t}))}
$$

for all $x, y \in M, t \leq 2 T$, where $B(y, \sqrt{t})$ denotes the geodesic ball centered at $y$ with radius $\sqrt{t}$.

A.2. For some fixed point $x_{0} \in M$, there exist $c_{x_{0}}>0, N \in \mathbf{N}$ such that

$$
m\left(B\left(x_{0}, r\right)\right) \leq c_{x_{0}} r^{N}, \quad r>0,
$$

where $m(d x)$ denotes for the Riemannian volume on $M$.

A.3. For any $r>0$,

$$
\inf _{x \in M} m(B(x, r))>0 .
$$


Remark 2.1. (i) Condition (A.1) is satisfied if, for example, the Ricci curvature is bounded from below, see [Stu92]. (A.3) holds if $M$ has bounded geometry. We refer the reader to [Dav89] for more details.

(ii) We note that (e.g. by the proof of [KLR03, Lemma 8.2]) (A.1)-(A.3) imply the conditions (C.1), (C.2), (C.3) in [KLR03] imposed there for one of the main results, namely Corollary 8.1 and Remark 8.3 in that paper, which we shall use below in a crucial way.

Let $\Gamma_{M}$ be the space of all $\mathbf{Z}_{+} \cup\{+\infty\}$-valued Radon measures on $M$. Equipped with the vague topology $\Gamma_{M}$ is a Polish space. The set of all $\gamma \in \Gamma_{M}$ such that $\gamma(\{x\}) \in\{0,1\}$ is called the configuration space over $M$. For simplicity, we also call $\Gamma_{M}$ configuration space over $M$. The geometry and analysis on configuration space has been developed in [AKR96a, AKR96b, AKR98a, AKR98b]. Let us recall some results and definitions from these papers. For $f \in C_{0}(M)$ (the space of all continuous functions on $M$ having compact support), set

$$
\langle f, \gamma\rangle:=\int_{M} f(x) \gamma(d x)=\sum_{x \in \gamma} f(x) .
$$

Define the space of smooth cylindrical functions on $\Gamma_{M}, \mathcal{F} C_{b}^{\infty}$, as the set of functions on $\Gamma_{M}$ of the form

$$
u(\gamma)=F\left(\left\langle f_{1}, \gamma\right\rangle, \ldots,\left\langle f_{n}, \gamma\right\rangle\right), \gamma \in \Gamma_{M}
$$

for some $n \in \mathbf{N}, F \in C_{b}^{\infty}\left(R^{n}\right)$, and $f_{1}, \ldots, f_{n} \in C_{0}^{\infty}(M)$. For $u$ as in (2.4) define its gradient $\nabla u$ as a mapping from $\Gamma_{M} \times M$ to $T M$ (the tangent bundle of $M)$ :

$$
\nabla u(\gamma, x):=\sum_{i=1}^{n} \partial_{i} F\left(\left\langle f_{1}, \gamma\right\rangle, \ldots,\left\langle f_{n}, \gamma\right\rangle\right) \nabla f_{i}(x), \gamma \in \Gamma_{M}, x \in M .
$$

Here $\partial_{i}$ denotes partial derivative with respect to the $i$-th coordinate, and $\nabla$ is the usual gradient on $M$.

Denote by $m$ the Riemannian volume on $M$. Let $\pi$ be the Poisson measure on $\Gamma_{M}$ with intensity $m$, i.e., the unique measure on $\Gamma_{M}$ whose Laplace transform is given by

$$
\int_{\Gamma_{M}} e^{\langle f, \gamma\rangle} \pi(d \gamma)=\exp \left(\int_{M}\left(e^{f(x)}-1\right) m(d x)\right)
$$

for all $f \in C_{0}(M)$. Introduce the pre-Dirichlet form:

$$
\begin{aligned}
\mathcal{E}_{0}(u, v):= & \int_{\Gamma_{M}}\langle\nabla u, \nabla v\rangle_{\gamma} \pi(d \gamma) \\
& u, v \in \mathcal{F} C_{b}^{\infty},
\end{aligned}
$$


where $\langle\nabla u, \nabla v\rangle_{\gamma}=\int_{M} \nabla u(\gamma, x) \cdot \nabla v(\gamma, x) \gamma(d x)$. It has been shown in [AKR98a] and [MR00] that $\mathcal{E}_{0}(u, v)=\int_{\Gamma_{M}}\langle\nabla u, \nabla v\rangle_{\gamma} \pi(d \gamma)$ is closable on $L^{2}\left(\Gamma_{M}, \pi\right)$ and its closure, denoted by $(\mathcal{E}, D(\mathcal{E}))$, is a quasi-regular Dirichlet form. Thus by the theory of Dirichlet forms (see [MR92]), there exists a diffusion process $\mathcal{M}:=\left\{\Omega, \mathcal{F}, \mathcal{F}_{t}, X_{t}, P_{\gamma}, \gamma \in \Gamma_{M}\right\}$ associated with the Dirichlet form $(\mathcal{E}, D(\mathcal{E}))$, where $\Omega:=C\left([0, \infty) \rightarrow \Gamma_{M}\right)$ is the canonical path space. The diffusion $\mathcal{M}$ itself is also called Brownian motion on the configuration space. It was also proved in [AKR98a] that starting with $\pi$ as initial distribution the process has the same law as the well known independent particle process (already studied by Doob in [Doo53]). Correspondingly, for $\gamma \in \Gamma_{M} P_{\gamma}$ should be the distribution of the process

$$
X_{t}^{\gamma}:=\sum_{x \in \gamma} \delta_{B_{t}^{x}}, t \geq 0
$$

where $\left(B_{t}^{x}\right)_{t \geq 0}$ are independent Brownian motions starting at $x \in \gamma$ and $\delta_{x}$ denotes Dirac measure at $x$. However, it is easy to see that this is not true for any $\gamma \in \Gamma_{M}$. For example, even when $M=\mathbf{R}, X_{t}=\sum_{i} \delta_{B_{t}^{i}}$ with initial configuration $\gamma=\sum_{i=1}^{\infty} \delta_{\log (i)}$ will not be a Radon measure on $\mathbf{R}$ for some $t>0$.

In [KLR03, Corollary 8.1 and Remark 8.3] it was proved that $\left(X_{t}\right)_{t \geq 0}$ defined in (2.6), however, a.s. does take values in $\Gamma_{M}$ for all $t \geq 0$ if one starts from points in a particular set $\Gamma_{\infty}$ and in fact $\left(X_{t}\right)_{t \geq 0}$ stays in $\Gamma_{\infty}$ for all $t \geq 0$. Let us recall the definition of $\Gamma_{\infty}$ from [KLR03]. First we fix a base point $x_{0}$ in $M$ once and for all. Let $d(x, y)$ denote the Riemannian distance on $M$. For each positive integer $m$, we define the functional

$$
B_{m}(\gamma):=\left\langle\exp \left[-\frac{1}{m} d\left(x_{0}, \cdot\right)\right], \gamma\right\rangle=\sum_{x \in \gamma} \exp \left[-\frac{1}{m} d\left(x_{0}, x\right)\right], \gamma \in \Gamma_{M},
$$

and define $\Gamma_{m}$ by

$$
\Gamma_{m}:=\left\{\gamma \in \Gamma_{M} \mid \gamma(\{x\}) \in\{0,1\} \text { for all } x \in M \text { and } B_{m}(\gamma)<\infty\right\} .
$$

Set

$$
\Gamma_{\infty}:=\bigcap_{m=1}^{\infty} \Gamma_{m}
$$

From now on we shall always use the version $\left(X_{t}^{\gamma}\right)_{t \geq 0}$ defined as in (2.6) above, for $\gamma \in \Gamma_{\infty}$, of the process $\mathcal{M}$ constructed by Dirichlet form methods. For 
topological reasons we shall, however, consider $\left(X_{t}^{\gamma}\right)_{t \geq 0}$ to take values in the bigger space $\Gamma_{M} \supset \Gamma_{\infty}$, since $\Gamma_{\infty}$ with the vague topology is not Polish.

In Section 8 , we shall present how our results on the sample path large deviations can be extended to other diffusions on $\Gamma_{M}$. Let us describe the latter here.

Let $\psi \in D(\mathcal{E})$ such that $0<\psi$ and $\int \psi^{2} d \pi=1$. As in [Ebe96] we define a new Dirichlet form on $L^{2}\left(\Gamma_{M}, \psi^{2} d \pi\right)$ by

$$
\begin{gathered}
\mathcal{E}_{\psi}(u, v):=\int_{\Gamma_{M}}\langle\nabla u, \nabla v\rangle_{\gamma} \psi^{2}(\gamma) \pi(d \gamma), \\
D\left(\mathcal{E}_{\psi}\right)=\overline{\mathcal{D}}^{\mathcal{E}_{\psi, 1},}
\end{gathered}
$$

where $\mathcal{E}_{\psi, 1}(u, u):=\mathcal{E}_{\psi}(u, u)+\int u^{2}(\gamma) \psi^{2}(\gamma) \pi(d \gamma)$ and $\mathcal{D}$ is given by

$$
\mathcal{D}:=\left\{u \in D(\mathcal{E}) \mid \int\left(\Gamma(u, u)(\gamma)+u^{2}(\gamma)\right) \psi^{2}(\gamma) \pi(d \gamma)<\infty\right\} .
$$

The diffusion process associated with the new Dirichlet form $\left(\mathcal{E}_{\psi}, D\left(\mathcal{E}_{\psi}\right)\right)$ will be denoted by $\mathcal{M}_{\psi}:=\left\{\Omega, \mathcal{F}, \mathcal{F}_{t}, X_{t}, Q_{\gamma}, \gamma \in \Gamma_{M}\right\}$, which are no longer independent Brownian particles.

For $u \in D\left(\mathcal{E}_{\psi}\right)$, set $\Gamma(u, u)(\gamma):=\langle\nabla u, \nabla u\rangle_{\gamma}$. Recall that the intrinsic metric of the Dirichlet form $\left(\mathcal{E}_{\psi}, D\left(\mathcal{E}_{\psi}\right)\right)$ is defined by

$$
\varrho(\gamma, \eta):=\sup \left\{u(\gamma)-u(\eta) \mid u \in D\left(\mathcal{E}_{\psi}\right) \cap C\left(\Gamma_{M}\right) \text { and } \Gamma(u, u) \leq 1\right\}
$$

for $\gamma, \eta \in \Gamma_{M}$. It was proved in [RS99] that $\varrho$ is a Wasserstein type distance on $\Gamma_{M}$ given by

$$
\varrho(\gamma, \eta):=\inf \left\{\sqrt{\int_{M \times M} \frac{1}{2} d(x, y)^{2} \omega(d x, d y)} \mid \omega \in \Gamma_{\gamma \times \eta}\right\},
$$

where $\Gamma_{\gamma \times \eta}$ denotes the set of $\omega \in \Gamma_{M \times M}$ having marginals $\gamma$ and $\eta$, and $d(x, y)$ stands for the Riemannian distance on $M$. We emphasize that $\varrho$ is independent of $\psi$.

\section{$\S 3 . \quad$ Some Preliminary Exponential Estimates}

From this section until the end of Section $7, \psi$ is assumed to be one.

Lemma 3.1. Let $T>0$. Then there exist $c_{1}(T)$ and $c_{2}(T)$ such that

$$
P\left(d\left(x, B_{t}^{x}\right)>r\right) \leq c_{1}(T) \exp \left(-\frac{c_{2}(T) r^{2}}{t}\right) \text {, for all } r>0, t \leq T, x \in M,
$$

where $B_{t}^{x}$ stands for the Brownian motion on $M$ starting from $x$. 
Proof. By condition A.1, for any $\delta>0$ there exists a constant $c_{1}(\delta)$ such that

$$
\frac{\exp \left[-\frac{d(x, y)^{2}}{(2-\delta) t}\right]}{c_{1}(\delta) m(B(y, \sqrt{t}))} \leq p_{t}(x, y) \leq c_{1}(\delta) \frac{\exp \left[-\frac{d(x, y)^{2}}{(2+\delta) t}\right]}{m(B(y, \sqrt{t}))}
$$

for all $x, y \in M, t \leq 2 T$.

Choose $\delta, \delta_{1}, \delta_{2} \in(0, \infty)$ such that $1 \leq a:=\frac{2+\delta}{2-\delta_{2}} \frac{1}{1-\delta_{1}} \leq 2$. It follows from (3.2) that

$$
\begin{aligned}
P\left(d\left(x, B_{t}^{x}\right)>r\right)= & \int_{d(x, y)>r} p_{t}(x, y) m(d y) \\
\leq & \int_{d(x, y)>r} c_{1}(\delta) \frac{\exp \left[-\frac{d(x, y)^{2}}{(2+\delta) t}\right]}{m(B(y, \sqrt{t}))} m(d y) \\
\leq & \exp \left[-\frac{\delta_{1} r^{2}}{(2+\delta) t}\right] \int_{d(x, y)>r} c_{1}(\delta) \frac{\exp \left[-\frac{\left(1-\delta_{1}\right) d(x, y)^{2}}{(2+\delta) t}\right]}{m(B(y, \sqrt{t}))} m(d y) \\
= & \exp \left[-\frac{\delta_{1} r^{2}}{(2+\delta) t}\right] \int_{d(x, y)>r} c_{1}(\delta) \frac{\exp \left[-\frac{d(x, y)^{2}}{\left(2-\delta_{2}\right) \hat{t}}\right]}{m(B(y, \sqrt{t}))} m(d y) \\
(\text { where } \tilde{t}:= & \left(\frac{2+\delta}{2-\delta_{2}} \frac{1}{\left.\left.1-\delta_{1}\right) t=a t \leq 2 T .\right)}\right. \\
\leq & \exp \left[-\frac{\delta_{1} r^{2}}{(2+\delta) t}\right] \int_{d(x, y)>r} c_{1}(\delta) c_{1}\left(\delta_{2}\right) \\
& \quad \times \frac{m(B(y, \sqrt{\tilde{t}}))}{m(B(y, \sqrt{t}))} \frac{1}{c_{1}\left(\delta_{2}\right)} \frac{\exp \left[-\frac{d(x, y)^{2}}{\left(2-\delta_{2}\right) \tilde{t}}\right]}{m(B(y, \sqrt{\tilde{t}}))} m(d y) \\
\leq & \quad c c_{1}(\delta) c_{1}\left(\delta_{2}\right) \exp \left[-\frac{\delta_{1} r^{2}}{(2+\delta) t}\right] \\
& \quad \times \exp [(d-1)(\sqrt{a}-1) \sqrt{t}] \int_{d(x, y)>r} p_{\tilde{t}}(x, y) m(d y) \\
\leq & c_{1} \exp \left[-\frac{\delta_{1} r^{2}}{(2+\delta) t}\right]
\end{aligned}
$$

where we have used the inequality:

$$
\frac{m(B(y, \sqrt{\tilde{t}}))}{m(B(y, \sqrt{t}))} \leq c \exp [(d-1)(\sqrt{a}-1) \sqrt{t}]
$$

which can be found, for example, in [GW00]. 
Corollary 3.2. Let $T>0$. Then there exists constants $c_{1}(T), c_{2}(T)>$ 0 such that for all $s \in(0, T]$

$$
P\left(\sup _{0 \leq t \leq s} d\left(x, B_{t}^{x}\right)>r\right) \leq c_{1}(T) \exp \left(-\frac{c_{2}(T) r^{2}}{s}\right) \text { for all } x \in M, r>0 .
$$

Proof. The corollary follows from Lemma 3.1 above and Lemma 8.1 in [KLR03].

\section{§4. Large Deviation Estimates for Finite Dimensional Projections: Upper Bounds}

Throughout this section, we fix a finite partition $D=\left\{0=t_{0}<t_{1}<t_{2}<\right.$ $\left.\cdots<t_{n}=1\right\}$ of $[0,1]$. Let $Y^{\varepsilon}$ be the random vector $Y^{\varepsilon}=\left(X_{\varepsilon t_{1}}, X_{\varepsilon t_{2}}, \ldots\right.$, $\left.X_{\varepsilon t_{n}}\right)$. Let $\mathcal{X}$ denote the set of all signed Radon measures on $M$. Equip $\mathcal{X}$ with the vague topology generated by

$$
\left\{U_{f, \alpha}=\{\nu \in \mathcal{X}||\langle f, \nu\rangle-\alpha \mid<\delta\}, f \in C_{0}(M), \alpha \in \mathbf{R}, \delta>0\right\} .
$$

$\mathcal{X}$ is a locally convex topological vector space with its topological dual $\mathcal{X}^{*}$ being identified as $C_{0}(M)$. It is well known that $\Gamma_{M}$ is a closed subspace of $\mathcal{X}$. Denote by $B\left(x_{0}, r\right)$ the geodesic ball $\left\{x \mid d\left(x_{0}, x\right)<r\right\}$ of $M$.

Lemma 4.1. For any $\delta>0$, there exists $c_{3}(\delta)>0$ such that for all $r>0, \varepsilon \leq 1, c>0$,

$$
E_{\gamma_{0}^{i}}\left[e^{\frac{1}{\varepsilon} c \chi_{B\left(x_{0}, r\right)}\left(B_{\varepsilon}\right)}\right] \leq 1+c_{3}(\delta) \exp \left[-\frac{\left(\left(d\left(\gamma_{0}^{i}, x_{0}\right)-r\right)^{2}-2(2+\delta) c\right)}{2(2+\delta) \varepsilon}\right]
$$

where $P_{\gamma_{0}^{i}}$ is the law of the Brownian motion on $M$ starting from $\gamma_{0}^{i}$.

Proof. By (3.2),

$$
\begin{aligned}
E_{\gamma_{0}^{i}}\left[e^{\frac{1}{\varepsilon} c \chi_{B\left(x_{0}, r\right)}\left(B_{\varepsilon}\right)}\right] & =e^{\frac{1}{\varepsilon} c} \int_{B\left(x_{0}, r\right)} p_{\varepsilon}\left(\gamma_{0}^{i}, y\right) m(d y)+\int_{B\left(x_{0}, r\right)^{c}} p_{\varepsilon}\left(\gamma_{0}^{i}, y\right) m(d y) \\
& \leq 1+e^{\frac{1}{\varepsilon} c} \int_{B\left(x_{0}, r\right)} c_{1}(\delta) \frac{\exp \left[-\frac{d\left(\gamma_{0}^{i}, y\right)^{2}}{(2+\delta) \varepsilon}\right]}{m(B(y, \sqrt{\varepsilon}))} m(d y) .
\end{aligned}
$$


Note that for $y \in B\left(x_{0}, r\right)$, we have $d\left(\gamma_{0}^{i}, y\right)>d\left(\gamma_{0}^{i}, x_{0}\right)-r$. Using the lower bound in (3.2) as in Lemma 3.1, we see that (4.2) is dominated by

$$
\begin{aligned}
1 & +e^{\frac{1}{\varepsilon} c} \exp \left[-\frac{\left(d\left(\gamma_{0}^{i}, x_{0}\right)-r\right)^{2}}{2(2+\delta) \varepsilon}\right] \int_{B\left(x_{0}, r\right)} c_{1}(\delta) \frac{\exp \left[-\frac{d\left(\gamma_{0}^{i}, y\right)^{2}}{2(2+\delta) \varepsilon}\right]}{m(B(y, \sqrt{\varepsilon}))} m(d y) \\
& \leq 1+e^{\frac{1}{\varepsilon} c} \exp \left[-\frac{\left(d\left(\gamma_{0}^{i}, x_{0}\right)-r\right)^{2}}{2(2+\delta) \varepsilon}\right] \int_{B\left(x_{0}, r\right)} c_{3}(\delta) p_{\tilde{\varepsilon}}\left(\gamma_{0}^{i}, y\right) m(d y) \\
& \leq 1+c_{3}(\delta) \exp \left[-\frac{\left(\left(d\left(\gamma_{0}^{i}, x_{0}\right)-r\right)^{2}-2(2+\delta) c\right)}{2(2+\delta) \varepsilon}\right],
\end{aligned}
$$

which proves the assertion.

Define $\mathcal{X}^{n}=\mathcal{X} \times \mathcal{X} \times \cdots \times \mathcal{X}$. Then it follows that

$$
\begin{aligned}
\left(\mathcal{X}^{n}\right)^{*} & =\mathcal{X}^{*} \oplus \mathcal{X}^{*} \oplus \cdots \oplus \mathcal{X}^{*} \\
& =C_{0}(M) \oplus C_{0}(M) \oplus \cdots \oplus C_{0}(M) .
\end{aligned}
$$

For $x, x_{1}, x_{2}, \ldots, x_{n} \in M$, set

$$
h_{x}\left(x_{1}, x_{2}, \ldots, x_{n}\right):=\frac{1}{2} \sum_{k=1}^{n} \frac{1}{\left(t_{k}-t_{k-1}\right)} d\left(x_{k}, x_{k-1}\right)^{2}, \text { where } x_{0}=x .
$$

Lemma 4.2. Let $F=\left(f_{1}, f_{2}, \ldots, f_{n}\right) \in\left(\mathcal{X}^{n}\right)^{*}$. Then

$$
\begin{aligned}
\Lambda(F) & :=\lim _{\varepsilon \rightarrow 0} \varepsilon \log P_{\gamma_{0}}\left[e^{\frac{1}{\varepsilon} F\left(Y^{\varepsilon}\right)}\right] \\
& =\int_{M} \sup _{\left(x_{1}, x_{2}, \ldots, x_{n}\right)}\left[\sum_{k=1}^{n} f_{k}\left(x_{k}\right)-h_{x}\left(x_{1}, x_{2}, \ldots, x_{n}\right)\right] \gamma_{0}(d x) .
\end{aligned}
$$

Proof. By the independence,

$$
\begin{aligned}
\Lambda(F) & =\lim _{\varepsilon \rightarrow 0} \varepsilon \log E_{\gamma_{0}}\left[e^{\frac{1}{\varepsilon} \sum_{k=1}^{n}\left\langle f_{k}, X_{\varepsilon t_{k}}\right\rangle}\right] \\
& =\lim _{\varepsilon \rightarrow 0} \varepsilon \log E_{\gamma_{0}}\left[e^{\frac{1}{\varepsilon} \sum_{k=1}^{n} \sum_{i=1}^{\infty} f_{k}\left(B_{\varepsilon t_{k}}^{i}\right)}\right] \\
& =\lim _{\varepsilon \rightarrow 0} \varepsilon \log E_{\gamma_{0}}\left[e^{\sum_{i=1}^{\infty} \frac{1}{\varepsilon} \sum_{k=1}^{n} f_{k}\left(B_{\varepsilon t_{k}}^{i}\right)}\right] \\
& =\lim _{\varepsilon \rightarrow 0} \varepsilon \log \Pi_{\gamma_{0}^{i} \in \gamma_{0}} E_{\gamma_{0}^{i}}\left[e^{\frac{1}{\varepsilon} \sum_{k=1}^{n} f_{k}\left(B_{\varepsilon t_{k}}^{i}\right)}\right] \\
& =\lim _{\varepsilon \rightarrow 0} \sum_{i} \varepsilon \log E_{\gamma_{0}^{i}}\left[e^{\frac{1}{\varepsilon} \sum_{k=1}^{n} f_{k}\left(B_{\varepsilon t_{k}}\right)}\right] .
\end{aligned}
$$


By the large deviation principle of Brownian motion (see, for example, [Aze80]), it follows that

$\lim _{\varepsilon \rightarrow 0} \varepsilon \log E_{\gamma_{0}^{i}}\left[e^{\frac{1}{\varepsilon} \sum_{k=1}^{n} f_{k}\left(B_{\varepsilon t_{k}}\right)}\right]=\sup _{\left(x_{1}, x_{2}, \ldots, x_{n}\right)}\left[\sum_{k=1}^{n} f_{k}\left(x_{k}\right)-h_{\gamma_{0}^{i}}\left(x_{1}, x_{2}, \ldots, x_{n}\right)\right]$.

Taking the limit inside the series in (4.5), we get

$$
\begin{aligned}
\Lambda(F) & =\sum_{i} \sup _{\left(x_{1}, x_{2}, \ldots, x_{n}\right)}\left[\sum_{k=1}^{n} f_{k}\left(x_{k}\right)-h_{\gamma_{0}^{i}}\left(x_{1}, x_{2}, \ldots, x_{n}\right)\right] \\
& =\int_{M} \sup _{\left(x_{1}, x_{2}, \ldots, x_{n}\right)}\left[\sum_{k=1}^{n} f_{k}\left(x_{k}\right)-h_{x}\left(x_{1}, x_{2}, \ldots, x_{n}\right)\right] \gamma_{0}(d x) .
\end{aligned}
$$

It now remains to justify that we can take the limit inside the series. We suppose that the supports of $f_{k}, 0 \leq k \leq n$, are contained in $B\left(x_{0}, r_{0}\right)$ for some $r_{0}$. By Lemma 4.1, we have

$$
\begin{aligned}
E_{\gamma_{0}^{i}}\left[e^{ \pm \frac{n}{\varepsilon} f_{k}\left(B_{\varepsilon t_{k}}\right)}\right] & \leq E_{\gamma_{0}^{i}}\left[e^{\frac{n}{\varepsilon}\left\|f_{k}\right\|_{\infty} \chi_{B\left(x_{0}, r_{0}\right)}\left(B_{\varepsilon t_{k}}\right)}\right] \\
& \leq 1+c_{3}(\delta) \exp \left[-\frac{\left(\left(d\left(\gamma_{0}^{i}, x_{0}\right)-r_{0}\right)^{2}-2(2+\delta) n t_{k}\left\|f_{k}\right\|_{\infty}\right)}{2(2+\delta) \varepsilon t_{k}}\right] .
\end{aligned}
$$

Hence by Schwartz's inequality,

$$
\begin{aligned}
& \left(E_{\gamma_{0}^{i}}\left[e^{\frac{n}{\varepsilon} f_{k}\left(B_{\varepsilon t_{k}}\right)}\right]\right)^{-1} \\
& \quad \leq E_{\gamma_{0}^{i}}\left[e^{-\frac{n}{\varepsilon} f_{k}\left(B_{\varepsilon t_{k}}\right)}\right] \\
& \quad \leq 1+c_{3}(\delta) \exp \left[-\frac{\left(\left(d\left(\gamma_{0}^{i}, x_{0}\right)-r_{0}\right)^{2}-2(2+\delta) n t_{k}\left\|f_{k}\right\|_{\infty}\right)}{2(2+\delta) \varepsilon t_{k}}\right] .
\end{aligned}
$$

Using Hölder's inequality we have that

$$
\begin{aligned}
& E_{\gamma_{0}^{i}}\left[e^{\frac{1}{\varepsilon} \sum_{k=1}^{n} f_{k}\left(B_{\varepsilon t_{k}}\right)}\right] \\
& \quad \leq \prod_{k=1}^{n}\left(1+c_{3}(\delta) \exp \left[-\frac{\left(\left(d\left(\gamma_{0}^{i}, x_{0}\right)-r_{0}\right)^{2}-2(2+\delta) n t_{k}\left\|f_{k}\right\|_{\infty}\right)}{2(2+\delta) \varepsilon t_{k}}\right]\right)^{\frac{1}{n}} .
\end{aligned}
$$

Similarly,

$$
\begin{aligned}
& \left(E _ { \gamma _ { 0 } ^ { i } } \left[e^{\left.\left.\frac{1}{\varepsilon} \sum_{k=1}^{n} f_{k}\left(B_{\varepsilon t_{k}}\right)\right]\right)^{-1}}\right.\right. \\
& \quad \leq \prod_{k=1}^{n}\left(1+c_{3}(\delta) \exp \left[-\frac{\left(\left(d\left(\gamma_{0}^{i}, x_{0}\right)-r_{0}\right)^{2}-2(2+\delta) n t_{k}\left\|f_{k}\right\|_{\infty}\right)}{2(2+\delta) \varepsilon t_{k}}\right]\right)^{\frac{1}{n}} .
\end{aligned}
$$


Hence it follows that for $\varepsilon \leq 1$,

$$
\begin{aligned}
\left|\log E_{\gamma_{0}^{i}}\left[e^{\frac{1}{\varepsilon} \sum_{k=1}^{n} f_{k}\left(B_{\varepsilon t_{k}}\right)}\right]\right| \\
\quad \leq \sum_{k=1}^{n} \frac{1}{n} \log \left(1+c_{3}(\delta) \exp \left[-\frac{\left(\left(d\left(\gamma_{0}^{i}, x_{0}\right)-r_{0}\right)^{2}-2(2+\delta) n t_{k}\left\|f_{k}\right\|_{\infty}\right)}{2(2+\delta) \varepsilon t_{k}}\right]\right) \\
\quad \leq \sum_{k=1}^{n} c_{3}(\delta) \frac{1}{n} \exp \left[-\frac{\left(\left(d\left(\gamma_{0}^{i}, x_{0}\right)-r_{0}\right)^{2}-2(2+\delta) n t_{k}\left\|f_{k}\right\|_{\infty}\right)}{2(2+\delta) \varepsilon t_{k}}\right] .
\end{aligned}
$$

Since $\sum_{i=1}^{\infty} \exp \left[-\frac{1}{m} d\left(x_{0}, \gamma_{0}^{i}\right)\right]<\infty$ for all $m$, the above estimates show that

$$
\sum_{i} \varepsilon \log E_{\gamma_{0}^{i}}\left[e^{\frac{1}{\varepsilon} \sum_{k=1}^{n} f_{k}\left(B_{\varepsilon t_{k}}\right)}\right]
$$

converges absolutely and uniformly with respect to $\varepsilon$, which justifies to take the limit inside the series.

Proposition 4.3. Let $\mu_{\varepsilon}$ be the law of $Y^{\varepsilon}$ on $\mathcal{X}^{n}$ under $P_{\gamma_{0}}$. Then $\left\{\mu_{\varepsilon}, \varepsilon \in(0,1]\right\}$ is exponentially tight, namely, for any $L>0$, there exists a compact subset $K_{L} \subset \mathcal{X}^{n}$ such that

$$
\limsup _{\varepsilon \rightarrow 0} \varepsilon \log P_{\gamma_{0}}\left(Y^{\varepsilon} \in K_{L}^{c}\right) \leq-L
$$

Proof. We first prove that the law of $X_{\varepsilon}$ is exponentially tight. So, let $\varepsilon \in(0,1]$. Note that a set of the form

$$
K_{\left\{L_{n}\right\}}=\bigcap_{n}\left\{\mu \in \mathcal{X}|| \mu \mid\left(B\left(x_{0}, n\right)\right) \leq L_{n}\right\}
$$

with $L_{n} \in(0, \infty)$, is relatively compact. Given $\hat{L}>0$, we will choose $L_{n}$ so that

$$
\limsup _{\varepsilon \rightarrow 0} \varepsilon \log P_{\gamma_{0}}\left(X_{\varepsilon} \in K_{\left\{L_{n}\right\}}^{c}\right) \leq-\hat{L}
$$

Let $m_{n}:=\sharp\left\{i \mid d\left(\gamma_{0}^{i}, x_{0}\right) \leq n+\sqrt{2(2+\delta)}\right\}$. By Lemma 4.1,

$$
E_{\gamma_{0}^{i}}\left[e^{\frac{1}{\varepsilon} \chi_{B\left(x_{0}, n\right)}\left(B_{\varepsilon}\right)}\right] \leq 1+c_{3} \exp \left[-\frac{\left(\left(d\left(\gamma_{0}^{i}, x_{0}\right)-n\right)^{2}-2(2+\delta)\right)}{2(2+\delta) \varepsilon}\right] .
$$


Hence for all $\varepsilon \leq 1$,

$$
\begin{aligned}
P_{\gamma_{0}}( & \left.X_{\varepsilon}\left(B\left(x_{0}, n\right)\right)>L_{n}\right) \\
\leq & e^{-\frac{L_{n}}{\varepsilon}} E_{\gamma_{0}}\left[e^{\left.\frac{1}{\varepsilon} \sum_{i=1}^{\infty} \chi_{B\left(x_{0}, n\right)}\left(B_{\varepsilon}^{i}\right)\right]}\right. \\
= & e^{-\frac{L_{n}}{\varepsilon}} \prod_{i=1}^{\infty} E_{\gamma_{0}^{i}}\left[e^{\frac{1}{\varepsilon} \chi_{B\left(x_{0}, n\right)}\left(B_{\varepsilon}^{i}\right)}\right] \\
\leq & e^{-\frac{L_{n}}{\varepsilon}} \prod_{i=1}^{\infty}\left(1+c_{3}(\delta) \exp \left[-\frac{\left(\left(d\left(\gamma_{0}^{i}, x_{0}\right)-n\right)^{2}-2(2+\delta)\right)}{2(2+\delta) \varepsilon}\right]\right) \\
\leq & e^{-\frac{L_{n}}{\varepsilon}} \prod_{\left(d\left(\gamma_{0}^{i}, x_{0}\right)-n\right)^{2}}\left(1+c_{3}(\delta) \exp \left[-\frac{\left(\left(d\left(\gamma_{0}^{i}, x_{0}\right)-n\right)^{2}-2(2+\delta)\right)}{2(2+\delta)>0}\right]\right) \\
& \times \quad \prod_{\left|d\left(\gamma_{0}^{i}, x_{0}\right)-n\right| \leq \sqrt{2(2+\delta)}}\left(1+c_{3}(\delta) \exp \left[\frac{1}{\varepsilon}\right]\right) \\
\leq & e^{-\frac{L_{n}}{\varepsilon}} \exp \left\{c_{3}(\delta) \sum_{i} e^{-\frac{\left(\left(d\left(\gamma_{0}^{i}, x_{0}\right)-n\right)^{2}-2(2+\delta)\right)}{2(2+\delta)}}\right\}\left(1+c_{3}(\delta) e^{\frac{1}{\varepsilon}}\right)^{m_{n}} \\
\leq & e^{-\frac{L_{n}}{\varepsilon}} \exp \left\{c_{3}(\delta) \sum_{i} e^{-\frac{\left(\left(d\left(\gamma_{0}^{i}, x_{0}\right)-n\right)^{2}-2(2+\delta)\right)}{2(2+\delta)}}\right\}\left(1+c_{3}(\delta)\right)^{m_{n}} e^{\frac{m_{n}}{\varepsilon}} \\
= & \exp \left\{-\frac{L_{n}-m_{n}}{\varepsilon}+c_{3}(\delta) \sum_{i} e^{-\frac{\left(\left(d\left(\gamma_{0}^{i}, x_{0}\right)-n\right)^{2}-2(2+\delta)\right)}{2(2+\delta)}}+m_{n} \log \left(1+c_{3}(\delta)\right)\right\} .
\end{aligned}
$$

Define

$$
L_{n}:=\hat{L}+m_{n}+c_{3}(\delta) \sum_{i} e^{-\frac{\left.\left(d\left(\gamma_{0}^{i}, x_{0}\right)-n\right)^{2}-2(2+\delta)\right)}{2(2+\delta)}}+m_{n} \log \left(1+c_{3}(\delta)\right)+n
$$

and define $K_{\hat{L}}:=K_{\left\{L_{n}\right\}}$ where $K_{\left\{L_{n}\right\}}$ is defined as in (4.8). Then we have

$$
\begin{aligned}
P_{\gamma_{0}}\left(X_{\varepsilon} \in\left(K_{\left\{L_{n}\right\}}^{c}\right)\right) & \leq \sum_{n=1}^{\infty} P_{\gamma_{0}}\left(X_{\varepsilon}\left(B\left(x_{0}, n\right)\right)>L_{n}\right) \\
& \leq \sum_{n=1}^{\infty} \exp \left\{-\frac{\hat{L}+n}{\varepsilon}\right\} \leq \frac{1}{e-1} e^{-\frac{\hat{L}}{\varepsilon}} .
\end{aligned}
$$

This implies (4.9).

Let $L>0$ and choose $\hat{L}$ such that $\inf _{1 \leq k \leq n}\left(\frac{\hat{L}}{t_{k}}\right)>L$. Let $K_{\hat{L}}$ be defined as above. Put

$$
K_{L}=\left(K_{\hat{L}}\right)^{n}
$$


Then $K_{L}$ is compact and

$$
\begin{aligned}
\limsup _{\varepsilon \rightarrow 0} \varepsilon \log P_{\gamma_{0}}\left(Y^{\varepsilon} \in K_{L}^{c}\right) & =\limsup _{\varepsilon \rightarrow 0} \varepsilon \log P_{\gamma_{0}}\left(\bigcup_{k=1}^{n}\left(X_{\varepsilon t_{k}} \in K_{\hat{L}}^{c}\right)\right) \\
& \leq \limsup _{\varepsilon \rightarrow 0} \varepsilon \log \left(n \max _{1 \leq k \leq n} P_{\gamma_{0}}\left(X_{\varepsilon t_{k}} \in K_{\hat{L}}^{c}\right)\right) \\
& =\max _{1 \leq k \leq n} \frac{1}{t_{k}}\left(\limsup _{\varepsilon \rightarrow 0} \varepsilon t_{k} \log P_{\gamma_{0}}\left(X_{\varepsilon t_{k}} \in K_{\hat{L}}^{c}\right)\right) \\
& \leq \max _{1 \leq k \leq n} \frac{1}{t_{k}}(-\hat{L}) \leq-L .
\end{aligned}
$$

Define, for $\left(\eta_{1}, \ldots, \eta_{n}\right) \in \mathcal{X}^{n}$,

$$
\begin{aligned}
I_{\gamma_{0}}\left(\eta_{1}, \ldots, \eta_{n}\right) & :=\sup _{\left(f_{1}, \ldots, f_{n}\right) \in C_{0}(M)^{n}}\left(\sum_{k=1}^{n}\left\langle f_{k}, \eta_{k}\right\rangle\right. \\
& \left.-\int_{M\left(x_{1}, x_{2}, \ldots, x_{n}\right)} \sup _{k=1}\left[\sum_{k}^{n} f_{k}\left(x_{k}\right)-h_{x}\left(x_{1}, x_{2}, \ldots, x_{n}\right)\right] \gamma_{0}(d x)\right) .
\end{aligned}
$$

Theorem 4.4. Let $\mu_{\varepsilon}$ be the law of $Y^{\varepsilon}$ on $\mathcal{X}^{n}$ under $P_{\gamma_{0}}$. Then, for any closed subset $F \subset \mathcal{X}^{n}$,

$$
\limsup _{\varepsilon \rightarrow 0} \varepsilon \log \mu_{\varepsilon}(F) \leq-\inf _{\left(\eta_{1}, \ldots, \eta_{n}\right) \in F} I_{\gamma_{0}}\left(\eta_{1}, \ldots, \eta_{n}\right) .
$$

Proof. The assertion follows by a combination of Lemma 4.2, Proposition 4.3 above and Theorem 4.5.20 in [DZ92].

\section{§5. Identification of the Rate Functional}

The main task of this section is to identify the rate function $I_{\gamma_{0}}\left(\eta_{1}, \ldots, \eta_{n}\right)$ as $\sum_{k=1}^{n} \frac{1}{\left(t_{k}-t_{k-1}\right)} \varrho\left(\eta_{k}, \eta_{k-1}\right)^{2}$ with $\eta_{0}=\gamma_{0}$. This turns out to be highly nontrivial. We will also show that the rate functional is good. Let $\mathcal{X}^{+}$denote the set of positive Radon measures on $M$. The definition of $\varrho$ in (2.11) extends to $\mathcal{X}^{+}$naturally with $\Gamma_{M}$ replaced by $\mathcal{X}^{+}$. Below, unless otherwise stated we fix $\gamma_{0} \in \mathcal{X}^{+}$. We also fix positive numbers $a_{1}, a_{2}, \ldots, a_{n}$. Introduce a functional $H\left(\gamma_{1}, \ldots, \gamma_{n}\right)$ on $\left(\mathcal{X}^{+}\right)^{n}$ by

$$
H\left(\gamma_{1}, \ldots, \gamma_{n}\right)=\sum_{k=1}^{n} a_{k} \varrho\left(\gamma_{k}, \gamma_{k-1}\right)^{2}
$$


Proposition 5.1. $\quad H$ is convex and lower semi-continuous on $\left(\mathcal{X}^{+}\right)^{n}$, equipped with the product topology of vague convergence.

Proof. Let $\gamma_{1}, \gamma_{2}, \bar{\gamma}_{1}, \bar{\gamma}_{2} \in \chi^{+}$. For any $0<\alpha<1$, we first show that

$$
\varrho\left(\alpha \gamma_{1}+(1-\alpha) \gamma_{2}, \alpha \bar{\gamma}_{1}+(1-\alpha) \bar{\gamma}_{2}\right)^{2} \leq \alpha \varrho\left(\gamma_{1}, \bar{\gamma}_{1}\right)^{2}+(1-\alpha) \varrho\left(\gamma_{2}, \bar{\gamma}_{2}\right)^{2} .
$$

To this end, we may and will assume $\varrho\left(\gamma_{1}, \bar{\gamma}_{1}\right)<\infty$ and $\varrho\left(\gamma_{2}, \bar{\gamma}_{2}\right)<\infty$. By Lemma 4.1 in [RS99] (although the Lemma was stated for $\Gamma_{M}$, its proof works also for $\left.\mathcal{X}^{+}\right)$, there exist $\eta, \bar{\eta} \in \mathcal{X}^{+}(M \times M)$ (the set of all positive Radon measures on $M \times M)$ with

$$
\begin{aligned}
\eta(d x, M) & =\gamma_{1}(d x), \eta(M, d y)=\bar{\gamma}_{1}(d y), \bar{\eta}(d x, M) \\
& =\gamma_{2}(d x), \bar{\eta}(M, d y)=\bar{\gamma}_{2}(d y)
\end{aligned}
$$

and such that

$$
\begin{aligned}
& \varrho\left(\gamma_{1}, \bar{\gamma}_{1}\right)^{2}=\frac{1}{2} \int_{M \times M} d(x, y)^{2} \eta(d x, d y) \\
& \varrho\left(\gamma_{2}, \bar{\gamma}_{2}\right)^{2}=\frac{1}{2} \int_{M \times M} d(x, y)^{2} \bar{\eta}(d x, d y) .
\end{aligned}
$$

Define $\eta^{*}=\alpha \eta+(1-\alpha) \bar{\eta}$. Then,

$$
\begin{aligned}
& \eta^{*}(d x, M)=\alpha \gamma_{1}(d x)+(1-\alpha) \gamma_{2}(d x) \\
& \eta^{*}(M, d y)=\alpha \bar{\gamma}_{1}(d y)+(1-\alpha) \bar{\gamma}_{2}(d y) .
\end{aligned}
$$

Thus,

$$
\begin{aligned}
\varrho\left(\alpha \gamma_{1}\right. & \left.+(1-\alpha) \gamma_{2}, \alpha \bar{\gamma}_{1}+(1-\alpha) \bar{\gamma}_{2}\right)^{2} \\
& \leq \int_{M \times M} d(x, y)^{2} \eta^{*}(d x, d y) \\
& =\alpha \int_{M \times M} d(x, y)^{2} \eta(d x, d y)+(1-\alpha) \int_{M \times M} d(x, y)^{2} \bar{\eta}(d x, d y) \\
& =\alpha \varrho\left(\gamma_{1}, \bar{\gamma}_{1}\right)^{2}+(1-\alpha) \varrho\left(\gamma_{2}, \bar{\gamma}_{2}\right)^{2},
\end{aligned}
$$

which proves $(5.2)$. Let $\vec{\gamma}_{1}=\left(\gamma_{1}^{1}, \ldots, \gamma_{n}^{1}\right), \vec{\gamma}_{2}=\left(\gamma_{1}^{2}, \ldots, \gamma_{n}^{2}\right) \in\left(\mathcal{X}^{+}\right)^{n}$. It follows from (5.5) that

$$
\begin{aligned}
H\left(\alpha \vec{\gamma}_{1}+(1-\alpha) \vec{\gamma}_{2}\right) & =\sum_{k=1}^{n} a_{k} \varrho\left(\alpha \gamma_{k}^{1}+(1-\alpha) \gamma_{k}^{2}, \alpha \gamma_{k-1}^{1}+(1-\alpha) \gamma_{k-1}^{2}\right)^{2} \\
& \leq \alpha \sum_{k=1}^{n} a_{k} \varrho\left(\gamma_{k}^{1}, \gamma_{k-1}^{1}\right)^{2}+(1-\alpha) \sum_{k=1}^{n} a_{k} \varrho\left(\gamma_{k}^{2}, \gamma_{k-1}^{2}\right)^{2} \\
& =\alpha H\left(\vec{\gamma}_{1}\right)+(1-\alpha) H\left(\vec{\gamma}_{2}\right),
\end{aligned}
$$


showing that $H(\cdot)$ is convex. Next we will prove that $H$ is lower semicontinuous. Let $\left\{\vec{\gamma}_{m}, m \geq 1\right\}$ be a sequence of elements in $\left(\mathcal{X}^{+}\right)^{n}$ converging vaguely to $\vec{\gamma}$. We need to show that

$$
H(\vec{\gamma}) \leq \liminf _{m \rightarrow \infty} H\left(\vec{\gamma}_{m}\right)
$$

For this purpose, we may and will assume that the $\operatorname{limit}_{\lim } \lim _{m \rightarrow \infty} H\left(\vec{\gamma}_{m}\right)$ exists and is finite as well as each $H\left(\vec{\gamma}_{m}\right)$. Write $\vec{\gamma}_{m}=\left(\gamma_{1}^{m}, \gamma_{2}^{m}, \ldots, \gamma_{n}^{m}\right)$ and $\vec{\gamma}=$ $\left(\gamma_{1}, \gamma_{2}, \ldots, \gamma_{n}\right)$. By Lemma 4.1 in [RS99] there exists $\eta_{k, k-1}^{m} \in \mathcal{X}^{+}(M \times M)$ with $\eta_{k, k-1}^{m}(d x, M)=\gamma_{k}^{m}(d x), \eta_{k, k-1}^{m}(M, d y)=\gamma_{k-1}^{m}(d y)$, such that

$$
\varrho\left(\gamma_{k}^{m}, \gamma_{k-1}^{m}\right)^{2}=\int_{M \times M} d(x, y)^{2} \eta_{k, k-1}^{m}(d x, d y) .
$$

Thus,

$$
\lim _{m \rightarrow \infty} H\left(\vec{\gamma}_{m}\right)=\lim _{m \rightarrow \infty} \sum_{k=1}^{n} a_{k} \int_{M \times M} d(x, y)^{2} \eta_{k, k-1}^{m}(d x, d y) .
$$

Let $\Pi_{1}$ be the projection operator from $M \times M$ to $M$ defined by $\Pi_{1}(x, y)=x$. For any compact set $K \subset M \times M$ and $k \geq 1$, we have

$$
\sup _{m} \eta_{k, k-1}^{m}(K) \leq \sup _{m} \prod_{1}^{*} \eta_{k, k-1}^{m}\left(\prod_{1}(K)\right)=\sup _{m} \gamma_{k}^{m}\left(\prod_{1}(K)\right)<\infty
$$

since $\gamma_{k}^{m}$ converges vaguely to $\gamma_{k}$ as $m \rightarrow \infty$. This implies that for each $1 \leq k \leq n$, the family $\left\{\eta_{k, k-1}^{m}, m \geq 1\right\}$ is relatively compact with respect to the topology of vague convergence. Now, choose a common subsequence $\left\{m_{l}\right\}$ such that

$$
\lim _{l \rightarrow \infty} \eta_{k, k-1}^{m_{l}}=\eta_{k, k-1}^{0}
$$

vaguely for each $1 \leq k \leq n$. Next we prove that

$$
\eta_{k, k-1}^{0}(d x, M)=\gamma_{k}(d x), \eta_{k, k-1}^{0}(M, d y)=\gamma_{k-1}(d y)
$$

Notice that this is not automatically a consequence of the vague convergence. We only prove one of them, say, $\eta_{k, k-1}^{0}(d x, M)=\gamma_{k}(d x)$, the other is proved analogously. Choose a sequence $\left\{\phi_{j}(y), j \geq 1\right\}$ of continuous functions on $M$ satisfying $0 \leq \phi_{j}(y) \leq 1, \phi_{j}(y)=1$ on $B\left(x_{0}, j\right), \phi_{j}(y)=0$ on $B\left(x_{0}, j+1\right)^{c}$. Take $f \in C_{0}(M)$. Suppose $\operatorname{supp}[f] \subset B\left(x_{0}, m_{0}\right)$ for some $m_{0}$. Then

$$
\begin{aligned}
\int_{M \times M} f(x) \eta_{k, k-1}^{0}(d x, d y) & =\lim _{j \rightarrow \infty} \int_{M \times M} f(x) \phi_{j}(y) \eta_{k, k-1}^{0}(d x, d y) \\
& =\lim _{j \rightarrow \infty} \lim _{l \rightarrow \infty} \int_{M \times M} f(x) \phi_{j}(y) \eta_{k, k-1}^{m_{l}}(d x, d y) .
\end{aligned}
$$


But,

$$
\begin{aligned}
\limsup _{l \rightarrow \infty} \mid & \int_{M \times M} f(x) \phi_{j}(y) \eta_{k, k-1}^{m_{l}}(d x, d y)-\int_{M \times M} f(x) \eta_{k, k-1}^{m_{l}}(d x, d y) \mid \\
& \leq \limsup _{l \rightarrow \infty} \int_{M \times M}|f(x)|\left(1-\phi_{j}(y)\right) \eta_{k, k-1}^{m_{l}}(d x, d y) \\
& \leq\|f\|_{\infty} \limsup _{l \rightarrow \infty} \int_{M \times M} \chi_{B\left(x_{0}, m_{0}\right)}(x) \chi_{B\left(x_{0}, j\right)^{c}}(y) \eta_{k, k-1}^{m_{l}}(d x, d y) \\
& \leq\|f\|_{\infty} \sup _{l} \int_{\left\{d^{2}(x, y) \geq\left(j-m_{0}\right)^{2}\right\}} \eta_{k, k-1}^{m_{l}}(d x, d y) \\
& \leq\|f\|_{\infty} \frac{1}{\left(j-m_{0}\right)^{2}} \sup _{l} \int_{M \times M} d^{2}(x, y) \eta_{k, k-1}^{m_{l}}(d x, d y) \\
& \leq \frac{M}{\left(j-m_{0}\right)^{2}}
\end{aligned}
$$

for some $M>0$. Combining (5.11) and (5.12) we arrive at

$$
\begin{aligned}
\int_{M \times M} f(x) \eta_{k, k-1}^{0}(d x, d y) & =\lim _{j \rightarrow \infty} \lim _{l \rightarrow \infty} \int_{M \times M} f(x) \phi_{j}(y) \eta_{k, k-1}^{m_{l}}(d x, d y) \\
& =\limsup _{l \rightarrow \infty} \int_{M \times M} f(x) \eta_{k, k-1}^{m_{l}}(d x, d y) \\
& =\lim _{l \rightarrow \infty} \int_{M} f(x) \gamma_{k}^{m_{l}}(d x) \\
& =\int_{M} f(x) \gamma_{k}(d x)
\end{aligned}
$$

Since $f$ was arbitrary, we conclude that $\eta_{k, k-1}^{0}(d x, M)=\gamma_{k}(d y)$.

Since $\int_{M \times M} d^{2}(x, y) \eta(d x, d y)$ is obviously lower semi-continuous with respect to $\eta$, it follows that

$$
\begin{aligned}
H(\vec{\gamma}) & \leq \sum_{k=1}^{n} a_{k} \int_{M \times M} d^{2}(x, y) \eta_{k, k-1}^{0}(d x, d y) \\
& \leq \sum_{k=1}^{n} a_{k} \liminf _{l \rightarrow \infty} \int_{M \times M} d^{2}(x, y) \eta_{k, k-1}^{m_{l}}(d x, d y) \\
& \leq \liminf _{l \rightarrow \infty} \sum_{k=1}^{n} a_{k} \int_{M \times M} d^{2}(x, y) \eta_{k, k-1}^{m_{l}}(d x, d y) \\
& =\lim _{l \rightarrow \infty} H\left(\vec{\gamma}_{m_{l}}\right) .
\end{aligned}
$$


Lemma 5.2. Let $f_{1}, f_{2}, \ldots, f_{n} \in C_{0}(M)$. Then

$$
g(x):=\sup _{x_{1}, x_{2}, \ldots, x_{n}}\left(\sum_{k=1}^{n} f_{k}\left(x_{k}\right)-h_{x}\left(x_{1}, x_{2}, \ldots, x_{n}\right)\right), x \in M,
$$

is lower semi-continuous and has compact support.

Proof. Clearly $g$ is lower semi-continuous. We need to show $g(x)=0$ outside some sufficiently big compact subset. Let $K$ be a compact subset of $M$ that contains the support of $f_{k}$ for all $1 \leq k \leq n$. We may only consider $x \in K^{c}$. For such $x$, we have that $g(x) \geq 0$ since $\sum_{k=1}^{n} f_{k}\left(x_{k}\right)-h_{x}\left(x_{1}, x_{2}, \ldots, x_{n}\right)=0$ for $x_{1}=x_{2}=\cdots=x_{n}=x$. Furthermore, denoting by $C$ the supremum of the function $\sum_{k=1}^{n} f_{k}(\cdot)$, we have

$$
\begin{aligned}
g(x) \leq & \sup _{\left(x_{1}, \ldots, x_{n}\right) \in\left(K^{c} \times \cdots \times K^{c}\right)^{c}}\left(\sum_{k=1}^{n} f_{k}\left(x_{k}\right)-h_{x}\left(x_{1}, x_{2}, \ldots, x_{n}\right)\right) \\
& \vee \sup _{\left(x_{1}, \ldots, x_{n}\right) \in K^{c} \times \cdots \times K^{c}}\left(-h_{x}\left(x_{1}, x_{2}, \ldots, x_{n}\right)\right) \\
\leq & \sup _{\left(x_{1}, \ldots, x_{n}\right) \in\left(K^{c} \times \cdots \times K^{c}\right)^{c}}\left(C-h_{x}\left(x_{1}, x_{2}, \ldots, x_{n}\right)\right) \vee 0 \\
\leq & \bigvee_{k=1}^{n} \sup _{\left(x_{1}, \ldots, x_{n}\right): x_{k} \in K}\left(C-h_{x}\left(x_{1}, x_{2}, \ldots, x_{n}\right)\right) \vee 0 .
\end{aligned}
$$

Thus, it suffices to prove that for each $1 \leq k \leq n$,

$$
g_{k}(x):=\sup _{\left(x_{1}, \ldots, x_{n}\right): x_{k} \in K}\left(C-h_{x}\left(x_{1}, x_{2}, \ldots, x_{n}\right)\right) \vee 0=0
$$

outside some compact subset. Since $x_{k} \in K$, we can find a compact subset $F_{k-1}$ such that for $x_{k-1} \in F_{k-1}^{c}$,

$$
C-h_{x}\left(x_{1}, x_{2}, \ldots, x_{n}\right) \leq C-\frac{1}{2} \frac{1}{t_{k}-t_{k-1}} d\left(x_{k}, x_{k-1}\right)^{2}<0 .
$$

Therefore, $g_{k}(x)$ can be written as

$$
g_{k}(x)=\sup _{\substack{\left(x_{1}, \ldots, x_{n}\right): x_{k} \in K \\ x_{k-1} \in F_{k-1}}}\left(C-h_{x}\left(x_{1}, x_{2}, \ldots, x_{n}\right)\right) \vee 0 .
$$

Repeating the same arguments, we can find compact subsets $F_{k-2}, F_{k-3}, \ldots, F_{1}$ such that

$$
\begin{aligned}
g_{k}(x)= & \sup _{\substack{\left(x_{1}, \ldots, x_{n}\right): x_{k} \in K, x_{k-1} \in F_{k-1}, \ldots, x_{1} \in F_{1}}}\left(C-h_{x}\left(x_{1}, x_{2}, \ldots, x_{n}\right)\right) \vee 0 \\
\leq & \sup _{x_{1} \in F_{1}}\left(C-\frac{1}{2} \frac{1}{t_{1}-t_{0}} d\left(x, x_{1}\right)^{2}\right) \vee 0 .
\end{aligned}
$$


The latter is clearly zero for $x$ outside some sufficiently big compact subset. This completes the proof of the lemma.

Lemma 5.3. Let $f_{1}, f_{2}, \ldots, f_{n} \in C_{0}(M)$. Then for $\gamma_{0} \in \Gamma_{M}$

$$
\begin{aligned}
\sup _{\gamma_{1}, \gamma_{2}, \ldots, \gamma_{n} \in \Gamma_{M}} & \left(\sum_{k=1}^{n}\left\langle f_{k}, \gamma_{k}\right\rangle-\sum_{k=1}^{n} \frac{1}{t_{k}-t_{k-1}} \varrho\left(\gamma_{k}, \gamma_{k-1}\right)^{2}\right) \\
& =\int_{M} \sup _{x_{1}, x_{2}, \ldots, x_{n}}\left(\sum_{k=1}^{n} f_{k}\left(x_{k}\right)-h_{x}\left(x_{1}, x_{2}, \ldots, x_{n}\right)\right) \gamma_{0}(d x) .
\end{aligned}
$$

Proof. Given $\gamma_{1}, \gamma_{2}, \ldots, \gamma_{n} \in \Gamma_{M}$ with $\varrho\left(\gamma_{k}, \gamma_{k-1}\right)<\infty, 1 \leq k \leq n$. Write $\gamma_{0}=\sum_{i=1}^{\infty} \delta_{\gamma_{0}^{i}}, \gamma_{k}=\sum_{i=1}^{\infty} \delta_{\gamma_{k}^{i}}$. Following [RS99, Lemma 4.1(iv)], renumbering if necessary, we may assume

$$
\varrho\left(\gamma_{k}, \gamma_{k-1}\right)^{2}=\frac{1}{2} \sum_{i=1}^{\infty} d\left(\gamma_{k}^{i}, \gamma_{k-1}^{i}\right)^{2} .
$$

Thus,

$$
\begin{aligned}
\sum_{k=1}^{n}\left\langle f_{k}, \gamma_{k}\right\rangle- & \sum_{k=1}^{n} \frac{1}{t_{k}-t_{k-1}} \varrho\left(\gamma_{k}, \gamma_{k-1}\right)^{2} \\
& =\sum_{i=1}^{\infty} \sum_{k=1}^{n} f_{k}\left(\gamma_{k}^{i}\right)-\sum_{i=1}^{\infty} \frac{1}{2} \sum_{k=1}^{n} \frac{1}{t_{k}-t_{k-1}} d\left(\gamma_{k}^{i}, \gamma_{k-1}^{i}\right)^{2} \\
& =\sum_{i=1}^{\infty}\left(\sum_{k=1}^{n} f_{k}\left(\gamma_{k}^{i}\right)-h_{\gamma_{0}^{i}}\left(\gamma_{1}^{i}, \gamma_{2}^{i}, \ldots, \gamma_{n}^{i}\right)\right) \\
& \leq \sum_{i=1}^{\infty} \sup _{x_{1}, x_{2}, \ldots, x_{n}}\left(\sum_{k=1}^{n} f_{k}\left(x_{k}\right)-h_{\gamma_{0}^{i}}\left(x_{1}, x_{2}, \ldots, x_{n}\right)\right) \\
& =\int_{M} \sup _{x_{1}, x_{2}, \ldots, x_{n}}\left(\sum_{k=1}^{n} f_{k}\left(x_{k}\right)-h_{x}\left(x_{1}, x_{2}, \ldots, x_{n}\right)\right) \gamma_{0}(d x)
\end{aligned}
$$

(Note that the last integral exists by Lemma 5.2)

Hence,

$$
\begin{aligned}
\sup _{\gamma_{1}, \gamma_{2}, \ldots, \gamma_{n} \in \Gamma_{M}} & \left(\sum_{k=1}^{n}\left\langle f_{k}, \gamma_{k}\right\rangle-\sum_{k=1}^{n} \frac{1}{t_{k}-t_{k-1}} \varrho\left(\gamma_{k}, \gamma_{k-1}\right)^{2}\right) \\
& \leq \int_{M} \sup _{x_{1}, x_{2}, \ldots, x_{n}}\left(\sum_{k=1}^{n} f_{k}\left(x_{k}\right)-h_{x}\left(x_{1}, x_{2}, \ldots, x_{n}\right)\right) \gamma_{0}(d x)
\end{aligned}
$$


To prove the dual inequality, we again set

$$
g(x):=\sup _{x_{1}, x_{2}, \ldots, x_{n}}\left(\sum_{k=1}^{n} f_{k}\left(x_{k}\right)-h_{x}\left(x_{1}, x_{2}, \ldots, x_{n}\right)\right) .
$$

Choose a compact subset $K$ containing the supports of $g$ and $f_{k}$ for all $1 \leq$ $k \leq n$. This is possible due to Lemma 5.2. Moreover, there exists an integer $N_{0}$ such that $\gamma_{0}^{i} \in K^{c}$ for $i>N_{0}$. Now, for any $\varepsilon>0$, there is $\left(x_{1}^{i}, \ldots, x_{n}^{i}\right) \in$ $M \times M \times \cdots \times M$ such that

$$
\begin{aligned}
\sum_{k=1}^{n} f_{k}\left(x_{k}^{i}\right)- & h_{\gamma_{0}^{i}}\left(x_{1}^{i}, x_{2}^{i}, \ldots, x_{n}^{i}\right) \\
& >\sup _{x_{1}, x_{2}, \ldots, x_{n}}\left(\sum_{k=1}^{n} f_{k}\left(x_{k}\right)-h_{\gamma_{0}^{i}}\left(x_{1}, x_{2}, \ldots, x_{n}\right)\right)-\frac{\varepsilon}{N_{0}}
\end{aligned}
$$

Define $\gamma_{k}^{i}:=x_{k}^{i}, i \leq N_{0}, \gamma_{k}^{i}:=\gamma_{0}^{i}, i>N_{0}$, and set $\gamma_{k}:=\sum_{i=1}^{\infty} \delta_{\gamma_{k}^{i}}$. We have setting $x_{0}^{i}:=\gamma_{0}^{i}$

$$
\begin{aligned}
\sum_{k=1}^{n}\left\langle f_{k}, \gamma_{k}\right\rangle & -\sum_{k=1}^{n} \frac{1}{t_{k}-t_{k-1}} \varrho\left(\gamma_{k}, \gamma_{k-1}\right)^{2} \\
& =\sum_{k=1}^{n} \sum_{i=1}^{N_{0}} f_{k}\left(\gamma_{k}^{i}\right)-\sum_{k=1}^{n} \frac{1}{t_{k}-t_{k-1}} \varrho\left(\gamma_{k}, \gamma_{k-1}\right)^{2} \\
& \geq \sum_{k=1}^{n} \sum_{i=1}^{N_{0}} f_{k}\left(x_{k}^{i}\right)-\sum_{k=1}^{n} \frac{1}{t_{k}-t_{k-1}} \frac{1}{2} \sum_{i=1}^{N_{0}} d\left(x_{k}^{i}, x_{k-1}^{i}\right)^{2} \\
& =\sum_{i=1}^{N_{0}}\left(\sum_{k=1}^{n} f_{k}\left(x_{k}^{i}\right)-h_{\gamma_{0}^{i}}\left(x_{1}^{i}, x_{2}^{i}, \ldots, x_{n}^{i}\right)\right) \\
& >\sum_{i=1}^{N_{0}} g\left(\gamma_{0}^{i}\right)-\varepsilon \\
& =\int_{M} g(x) \gamma_{0}(d x)-\varepsilon .
\end{aligned}
$$

Since $\varepsilon$ is arbitrary, the dual inequality follows and the lemma is proved.

Proposition 5.4. $\quad I_{\gamma_{0}}\left(\gamma_{1}, \gamma_{2}, \ldots, \gamma_{n}\right)=\sum_{k=1}^{n} \frac{1}{t_{k}-t_{k-1}} \varrho\left(\gamma_{k}, \gamma_{k-1}\right)^{2}$ for all $\gamma_{0}, \gamma_{1}, \ldots, \gamma_{n} \in \Gamma_{M}$. 
Proof. By Lemma 5.3,

$$
\begin{aligned}
& I_{\gamma_{0}}\left(\gamma_{1}, \gamma_{2}, \ldots, \gamma_{n}\right)=\sup _{\left(f_{1}, \ldots, f_{n}\right) \in C_{0}(M)^{n}}\left(\sum_{k=1}^{n}\left\langle f_{k}, \gamma_{k}\right\rangle\right. \\
&\left.-\sup _{\tilde{\gamma}_{1}, \tilde{\gamma}_{2}, \ldots, \tilde{\gamma}_{n} \in \Gamma_{M}}\left(\sum_{k=1}^{n}\left\langle f_{k}, \tilde{\gamma}_{k}\right\rangle-\sum_{k=1}^{n} \frac{1}{t_{k}-t_{k-1}} \varrho\left(\tilde{\gamma}_{k}, \tilde{\gamma}_{k-1}\right)^{2}\right)\right) .
\end{aligned}
$$

Since for $f_{1}, \ldots, f_{n} \in C_{0}(M)$

$$
\begin{aligned}
\sum_{k=1}^{n}\left\langle f_{k}, \gamma_{k}\right\rangle & -\sup _{\tilde{\gamma}_{1}, \tilde{\gamma}_{2}, \ldots, \tilde{\gamma}_{n} \in \Gamma_{M}}\left(\sum_{k=1}^{n}\left\langle f_{k}, \tilde{\gamma}_{k}\right\rangle-\sum_{k=1}^{n} \frac{1}{t_{k}-t_{k-1}} \varrho\left(\tilde{\gamma}_{k}, \tilde{\gamma}_{k-1}\right)^{2}\right) \\
& \leq \sum_{k=1}^{n} \frac{1}{t_{k}-t_{k-1}} \varrho\left(\gamma_{k}, \gamma_{k-1}\right)^{2},
\end{aligned}
$$

we have

$$
I_{\gamma_{0}}\left(\gamma_{1}, \gamma_{2}, \ldots, \gamma_{n}\right) \leq \sum_{k=1}^{n} \frac{1}{t_{k}-t_{k-1}} \varrho\left(\gamma_{k}, \gamma_{k-1}\right)^{2} .
$$

On the other hand, by the general theorem on the inverse Legendre transform of convex functions (see [DZ92, Lemma 4.5.8]) and Proposition 5.1, 5.3, we see that

$$
\begin{aligned}
& \sum_{k=1}^{n} \frac{1}{t_{k}-t_{k-1}} \varrho\left(\gamma_{k}, \gamma_{k-1}\right)^{2}= \sup _{\left(f_{1}, \ldots, f_{n}\right) \in C_{0}(M)^{n}}\left\{\sum_{k=1}^{n}\left\langle f_{k}, \gamma_{k}\right\rangle\right. \\
&\left.-\sup _{\tilde{\gamma}_{1}, \tilde{\gamma}_{2}, \ldots, \tilde{\gamma}_{n} \in \chi^{+}(M)}\left(\sum_{k=1}^{n}\left\langle f_{k}, \tilde{\gamma}_{k}\right\rangle-\sum_{k=1}^{n} \frac{1}{t_{k}-t_{k-1}} \varrho\left(\tilde{\gamma}_{k}, \tilde{\gamma}_{k-1}\right)^{2}\right)\right\}
\end{aligned}
$$

which is smaller than $I_{\gamma_{0}}\left(\gamma_{1}, \gamma_{2}, \ldots, \gamma_{n}\right)$.

Proposition 5.5. For any $\gamma_{0} \in \Gamma_{M}$ the rate functional $I_{\gamma_{0}}$ is good on $\Gamma_{M}^{n}$, i.e., for any $L>0$ the level set

$$
R_{L}:=\left\{\left(\gamma_{1}, \ldots, \gamma_{n}\right) \in \Gamma_{M}^{n} \mid I_{\gamma_{0}}\left(\gamma_{1}, \gamma_{2}, \ldots, \gamma_{n}\right) \leq L\right\}
$$

is compact in $\Gamma_{M}^{n}$.

Proof. By Proposition 5.1 the rate functional $I_{\gamma_{0}}$ is lower semi-continuous on $\Gamma_{M}^{n}$. So, we only need to show that every level set is relatively compact in $\Gamma_{M}^{n}$. So, let $L>0$. It is sufficient to prove that for each $1 \leq k \leq n$ the set

$$
R_{L}^{k}:=\Pi_{k}\left(R_{L}\right)
$$


is relatively compact, where $\Pi_{k}: \Gamma_{M}^{n} \rightarrow \Gamma_{M}$ is the natural projection on the $k$-th component. Let $r_{0}>0, \gamma \in \Gamma_{M}$. Then for any $\omega \in \Gamma_{\gamma_{0} \times \gamma}$,

$$
\begin{aligned}
\gamma\left(B\left(x_{0}, r_{0}\right)\right)= & \int_{M \times M} \chi_{B\left(x_{0}, r_{0}\right)}(y) \omega(d x, d y) \\
= & \int_{M \times M} \chi_{B\left(x_{0}, r_{0}+\sqrt{2}\right)}(x) \chi_{B\left(x_{0}, r_{0}\right)}(y) \omega(d x, d y) \\
& +\int_{M \times M} \chi_{B\left(x_{0}, r_{0}+\sqrt{2}\right)^{c}}(x) \chi_{B\left(x_{0}, r_{0}\right)}(y) \omega(d x, d y) \\
\leq & \int_{M \times M} \chi_{B\left(x_{0}, r_{0}+\sqrt{2}\right)}(x) \omega(d x, d y) \\
& +\frac{1}{2} \int_{M \times M} d(x, y)^{2} \omega(d x, d y) \\
= & \gamma_{0}\left(B\left(x_{0}, r_{0}+\sqrt{2}\right)\right)+\frac{1}{2} \int_{M \times M} d(x, y)^{2} \omega(d x, d y) .
\end{aligned}
$$

Taking the infimum over $\omega$ we get

$$
\gamma\left(B\left(x_{0}, r_{0}\right)\right) \leq \gamma_{0}\left(B\left(x_{0}, r_{0}+\sqrt{2}\right)\right)+\varrho\left(\gamma_{0}, \gamma\right)^{2} .
$$

If $\gamma=\gamma_{k} \in R_{L}^{k}$, then by definition there exist $\gamma_{1}, \ldots, \gamma_{k-1}, \gamma_{k+1}, \ldots, \gamma_{n} \in \Gamma_{M}$ so that $\left(\gamma_{1}, \ldots, \gamma_{n}\right) \in R_{L}$. Hence $\left(\right.$ since $\sum_{l=1}^{n}\left(t_{l}-t_{l-1}\right)=1$ )

$$
\varrho\left(\gamma_{0}, \gamma_{k}\right)^{2} \leq\left(\sum_{l=1}^{n} \varrho\left(\gamma_{l}, \gamma_{l-1}\right)\right)^{2} \leq \sum_{l=1}^{n} \frac{1}{t_{l}-t_{l-1}} \varrho\left(\gamma_{l}, \gamma_{l-1}\right)^{2} \leq L
$$

Thus,

$$
\sup _{\gamma_{k} \in R_{L}^{k}} \gamma_{k}\left(B\left(x_{0}, r_{0}\right)\right) \leq \gamma_{0}\left(B\left(x_{0}, r_{0}+\sqrt{2}\right)\right)+L
$$

Since $r_{0}$ was arbitrary, this implies that $R_{L}^{k}$ is relatively compact in $\mathcal{X}^{+}$. Since $\Gamma_{M}$ is Polish, it is closed in $\mathcal{X}^{+}$, so the assertion follows.

\section{§6. Large Deviation Estimates for Finite Dimensional Projections: the Lower Bounds}

The lower bound holds under even a little stronger topology on $\Gamma_{M}$ which was introduced in [KLR03], namely, the topology on $\Gamma_{\infty}$ induced by the following metric:

$$
d_{\infty}\left(\gamma_{1}, \gamma_{2}\right):=d_{v}\left(\gamma_{1}, \gamma_{2}\right)+\sum_{m=1}^{\infty} 2^{-m} \frac{\left|B_{m}\left(\gamma_{1}\right)-B_{m}\left(\gamma_{2}\right)\right|}{\left(1+\left|B_{m}\left(\gamma_{1}\right)-B_{m}\left(\gamma_{2}\right)\right|\right)}
$$


where $d_{v}$ is any metric compatible with the vague topology. Let $U$ be an $d_{v}$-open neighborhood in $\Gamma_{M}$ described by

$$
U=\left\{\gamma \in \Gamma_{M}\left|\gamma\left(\partial W_{r}\right)=0, \gamma\right|_{W_{r}}=\sum_{i=1}^{n} \delta_{x_{i}} \text { with } \sum_{i=1}^{n} d\left(x_{i}, y_{i}\right)^{2}<\delta_{0}\right\}
$$

where $W_{r}:=B\left(x_{0}, r\right), r>0, y_{1}, \ldots, y_{n}$ are fixed points in $W_{r}$, and $\delta_{0}>0$.

Proposition 6.1. Let $\gamma_{0} \in \Gamma_{\infty}$. Then, for any $\delta_{1}>0$ and distinct integers $i_{1}, \ldots, i_{n}$,

$$
\begin{aligned}
& \text { (6.3) } \liminf _{\varepsilon \rightarrow 0, \gamma \rightarrow \gamma_{0}} \varepsilon \log P_{\gamma}\left(X_{\varepsilon} \in U\right) \geq-\frac{1}{2} \sum_{j=1}^{n} d\left(y_{j}, \gamma_{0}^{i_{j}}\right)^{2}\left(1+\delta_{1}\right)-\frac{1}{2}\left(\frac{1}{\delta_{1}}+1\right) \delta_{0} \\
& -\sum_{k \notin\left\{i_{1}, i_{2}, \ldots, i_{n}\right\}} \frac{1}{2} d\left(\gamma_{0}^{k}, \bar{W}_{r}^{c}\right)^{2},
\end{aligned}
$$

where $d\left(\gamma_{0}^{k}, \bar{W}_{r}^{c}\right)$ denotes the distance from $\gamma_{0}^{k}$ to $\bar{W}_{r}^{c}$ and $\gamma \rightarrow \gamma_{0}$ with respect to $d_{\infty}$, in $\Gamma_{\infty}$.

Proof. Let $\left\{\gamma_{m}, m \geq 1\right\}$ be any sequence in $\Gamma_{\infty}$ such that $d_{\infty}\left(\gamma_{m}, \gamma_{0}\right) \rightarrow 0$. It is sufficient to show (6.3) for such a sequence. Write $\gamma_{m}=\sum_{i=1}^{\infty} \delta_{\gamma_{m}^{i}}$ and $\gamma_{0}=\sum_{i=1}^{\infty} \delta_{\gamma_{0}^{i}}$. Rearranging elements in $\gamma_{m}$ if necessary, we may assume that $\lim _{m \rightarrow \infty} \gamma_{m}^{i}=\gamma_{0}^{i}$ for $i \geq 1$ (see the proof of Theorem 6.1 in [KLR03]). For distinct integers $i_{1}, i_{2}, i_{3}, \ldots, i_{n}$, define

$$
\begin{aligned}
A_{i_{1}, i_{2}, \ldots, i_{n}}:=\left\{B_{\varepsilon}^{i_{1}} \in W_{r}, \ldots, B_{\varepsilon}^{i_{n}} \in W_{r}, \sum_{j=1}^{n} d\left(B_{\varepsilon}^{i_{j}}, y_{j}\right)^{2}<\delta_{0}\right. \\
\left.B_{\varepsilon}^{k} \in \bar{W}_{r}^{c}, k \notin\left\{i_{1}, \ldots, i_{n}\right\}\right\} .
\end{aligned}
$$

Then

$$
\left\{X_{\varepsilon} \in U\right\}=\bigcup_{i_{1}, i_{2}, \ldots, i_{n}} A_{i_{1}, i_{2}, \ldots, i_{n}}
$$

hence,

$$
P_{\gamma_{m}}\left(X_{\varepsilon} \in U\right)=\sum_{i_{1}, i_{2}, \ldots, i_{n}}^{\infty} P_{\gamma_{m}}\left(A_{i_{1}, i_{2}, \ldots, i_{n}}\right)
$$


So, for any distinct integers $i_{1}, i_{2}, i_{3}, \ldots, i_{n}$ we have

$$
\begin{aligned}
& P_{\gamma_{m}}\left(X_{\varepsilon} \in U\right) \geq P_{\gamma_{m}}\left(A_{i_{1}, i_{2}, \ldots, i_{n}}\right)
\end{aligned}
$$

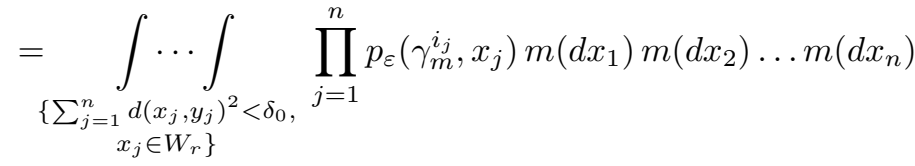

$$
\begin{aligned}
& \times \prod_{k \notin\left\{i_{1}, i_{2}, \ldots, i_{n}\right\}} P_{\gamma_{m}^{k}}\left(B_{\varepsilon} \in \bar{W}_{r}^{c}\right) \\
& =a_{\varepsilon}^{i_{1}, i_{2}, \ldots, i_{n}} \times b_{\varepsilon}^{i_{1}, i_{2}, \ldots, i_{n}} .
\end{aligned}
$$

Note that for any $\delta_{1}>0$,

$$
\sum_{j=1}^{n} d\left(x_{j}, \gamma_{m}^{i_{j}}\right)^{2} \leq\left(1+\frac{1}{\delta_{1}}\right) \sum_{j=1}^{n} d\left(x_{j}, y_{j}\right)^{2}+\left(1+\delta_{1}\right) \sum_{j=1}^{n} d\left(y_{j}, \gamma_{m}^{i_{j}}\right)^{2} .
$$

This and (3.2) imply

$$
\begin{aligned}
a_{\varepsilon}^{i_{1}, i_{2}, \ldots, i_{n}} \geq & \int_{\substack{\left\{\sum_{j=1}^{n} d^{2}\left(x_{j}, y_{j}\right)<\delta_{0}, x_{j} \in W_{r}\right\}}} \ldots \prod_{j=1}^{n} \frac{\exp \left[-\frac{d\left(\gamma_{m}^{\left.i_{j}, x_{j}\right)^{2}}\right.}{(2-\delta) \varepsilon}\right]}{c_{1}(\delta) m\left(B\left(\gamma_{m}^{i_{j}}, \sqrt{\varepsilon}\right)\right)} m\left(d x_{1}\right) \ldots m\left(d x_{n}\right) \\
& \geq \int_{\substack{\left\{\sum_{j=1}^{n} d^{2}\left(x_{j}, y_{j}\right)<\delta_{0}, x_{j} \in W_{r}\right\}}} \exp \left(-\frac{\left(1+\frac{1}{\delta_{1}}\right) \delta_{0}}{(2-\delta) \varepsilon}\right) \prod_{j=1}^{n} \frac{1}{c_{1}(\delta) m\left(B\left(\gamma_{m}^{i_{j}}, \sqrt{\varepsilon}\right)\right)} \\
& \times \exp \left(-\left(1+\delta_{1}\right) \frac{\sum_{j=1}^{n} d\left(y_{j}, \gamma_{m}^{i_{j}}\right)^{2}}{(2-\delta) \varepsilon}\right) m\left(d x_{1}\right) m\left(d x_{2}\right) \ldots m\left(d x_{n}\right) .
\end{aligned}
$$

Since $\lim _{\varepsilon \rightarrow 0, m \rightarrow \infty} \varepsilon \log \left(\prod_{j=1}^{n} \frac{1}{c_{1}(\delta) m\left(B\left(\gamma_{m}^{i_{j}}, \sqrt{\varepsilon}\right)\right)}\right)=0$, we have

$\liminf _{\varepsilon \rightarrow 0, m \rightarrow \infty} \varepsilon \log a_{\varepsilon}^{i_{1}, i_{2}, \ldots, i_{n}} \geq-\frac{1}{2-\delta}\left(\frac{1}{\delta_{1}}+1\right) \delta_{0}-\left(1+\delta_{1}\right) \frac{1}{2-\delta} \sum_{j=1}^{n} d\left(y_{j}, \gamma_{0}^{i_{j}}\right)^{2}$.

To treat $b_{\varepsilon}^{i_{1}, i_{2}, \ldots, i_{n}}$, we need the following.

Lemma 6.2. Let $c_{1}(1), c_{2}(1)$ be as in Lemma $3.1($ for $T:=1)$. Then

$$
\left|\log P\left(d\left(x_{0}, B_{\varepsilon}^{x}\right)>r\right)\right| \leq \frac{c_{1}(1) \exp \left(-c_{2}(1) d\left(x_{0}, x\right)^{2}\right)}{1-c_{1}(1) \exp \left(-c_{2}(1) d\left(x_{0}, x\right)^{2}\right)}
$$

for all $r>0$ and $x \in M$ with $d\left(x_{0}, x\right)>2 r$ and $\varepsilon \leq 1$. 
Proof. Note that $d\left(x_{0}, B_{\varepsilon}^{x}\right)>d\left(x_{0}, x\right)-d\left(x, B_{\varepsilon}^{x}\right)$. Using $d\left(x_{0}, x\right)>2 r$ and Lemma 3.1 it follows that

$$
\begin{aligned}
P\left(d\left(x_{0}, B_{\varepsilon}^{x}\right)>r\right) & \geq P\left(d\left(x, B_{\varepsilon}^{x}\right) \leq \frac{1}{2} d\left(x_{0}, x\right)\right) \\
& =1-P\left(d\left(x, B_{\varepsilon}^{x}\right)>\frac{1}{2} d\left(x_{0}, x\right)\right) \\
& \geq 1-c_{1}(1) \exp \left(-c_{2}(1) d\left(x_{0}, x\right)^{2}\right) .
\end{aligned}
$$

Hence,

$$
\begin{aligned}
\left|\log P\left(d\left(x_{0}, B_{\varepsilon}^{x}\right)>r\right)\right| & =-\log P\left(d\left(x_{0}, B_{\varepsilon}^{x}\right)>r\right) \\
& \leq \log \left(\frac{1}{1-c_{1}(1) \exp \left(-c_{2}(1) d\left(x_{0}, x\right)^{2}\right)}\right) \\
& =\log \left(1+\frac{c_{1}(1) \exp \left(-c_{2}(1) d\left(x_{0}, x\right)^{2}\right)}{1-c_{1}(1) \exp \left(-c_{2}(1) d\left(x_{0}, x\right)^{2}\right)}\right) \\
& \leq \frac{c_{1}(1) \exp \left(-c_{2}(1) d\left(x_{0}, x\right)^{2}\right)}{1-c_{1}(1) \exp \left(-c_{2}(1) d\left(x_{0}, x\right)^{2}\right)},
\end{aligned}
$$

which proves the assertion.

Corollary 6.3. We have

$$
\lim _{\varepsilon \rightarrow 0, m \rightarrow \infty} \varepsilon \log b_{\varepsilon}^{i_{1}, i_{2}, \ldots, i_{n}}=-\frac{1}{2} \sum_{k \notin\left\{i_{1}, \ldots, i_{n}\right\}} d\left(\gamma_{0}^{k}, \bar{W}_{r}^{c}\right)^{2} .
$$

Proof. Note that

$$
\varepsilon \log b_{\varepsilon}^{i_{1}, i_{2}, \ldots, i_{n}}=\sum_{k \notin\left\{i_{1}, \ldots, i_{n}\right\}} \varepsilon \log P_{\gamma_{m}^{k}}\left(d\left(x_{0}, B_{\varepsilon}\right)>r\right) .
$$

By the large deviation principle of Brownian motion,

$$
\lim _{\varepsilon \rightarrow 0, m \rightarrow \infty} \varepsilon \log P_{\gamma_{m}^{k}}\left(d\left(x_{0}, B_{\varepsilon}\right)>r\right)=-\frac{1}{2} d\left(\gamma_{0}^{k}, \bar{W}_{r}^{c}\right)^{2} .
$$

Since $\gamma_{0} \in \Gamma_{\infty}$ and $\gamma_{m} \rightarrow \gamma_{0}$ with respect to $d_{\infty}$, (6.5) now follows from Lemma 6.2 and the dominated convergence theorem.

Using Corollary 6.3 and letting $\delta \rightarrow 0$ we get Proposition 6.1.

Proposition 6.4. Let $\gamma_{0} \in \Gamma_{\infty}$. Suppose that $O \subset \Gamma_{\infty}$ is an open subset w.r.t. $d_{\infty}$. Then,

$$
\liminf _{\varepsilon \rightarrow 0, \gamma \rightarrow \gamma_{0}} \varepsilon \log P_{\gamma}\left(X_{\varepsilon} \in O\right) \geq-\inf _{\gamma \in O} \varrho\left(\gamma_{0}, \gamma\right)^{2},
$$

where $\gamma \rightarrow \gamma_{0}$ with respect to $d_{\infty}$ in $\Gamma_{\infty}$. 
We first prove the analogue of Proposition 6.4 for the vague topology.

Proposition 6.5. Let $\gamma_{0} \in \Gamma_{\infty}$. Suppose that $O \subset \Gamma_{M}$ is an open subset w.r.t. the vague topology. Then,

$$
\liminf _{\varepsilon \rightarrow 0, \gamma \rightarrow \gamma_{0}} \varepsilon \log P_{\gamma}\left(X_{\varepsilon} \in O\right) \geq-\inf _{\gamma \in O} \varrho\left(\gamma_{0}, \gamma\right)^{2},
$$

where $\gamma \rightarrow \gamma_{0}$ with respect to $d_{\infty}$ in $\Gamma_{\infty}$.

Proof. It is sufficient to prove

$$
\liminf _{\varepsilon \rightarrow 0, \gamma \rightarrow \gamma_{0}} \varepsilon \log P_{\gamma}\left(X_{\varepsilon} \in O\right) \geq-\varrho\left(\gamma_{0}, \hat{\gamma}\right)^{2}
$$

for any $\hat{\gamma} \in O$. So, fix $\hat{\gamma} \in O$. Without loss of generality, we can assume that

$$
\varrho\left(\gamma_{0}, \hat{\gamma}\right)^{2}=\frac{1}{2} \sum_{i=1}^{\infty} d\left(\hat{\gamma}^{i}, \gamma_{0}^{i}\right)^{2}<\infty
$$

Choose an increasing sequence $W_{r_{n}}$ of geodesic balls such that $\bigcup W_{r_{n}}=M$ and

$$
\left.\hat{\gamma}\right|_{W_{r_{n}}}=\sum_{i=1}^{m_{n}} \delta_{\hat{\gamma}^{i}}, \hat{\gamma}\left(\partial W_{r_{n}}\right)=0 .
$$

Let $\delta_{l}, l \geq 1$, be a sequence of positive numbers converging to zero. Set

$$
U_{n, l}:=\left\{\gamma \in \Gamma_{M}\left|\gamma\left(\partial W_{r_{n}}\right)=0, \gamma\right|_{W_{r_{n}}}=\sum_{i=1}^{m_{n}} \delta_{x_{i}} \text { with } \sum_{i=1}^{m_{n}} d\left(x_{i}, \hat{\gamma}^{i}\right)^{2}<\delta_{l}\right\} .
$$

Obviously, $\left\{U_{n, m} \mid n, m \in \mathbf{N}\right\}$ form a basis of neighbourhoods for $\hat{\gamma}$ in the vague topology. Since $O$ is open, there exist $n_{0}, l_{0}$ such that $U_{n, l} \subset O$ for $n \geq n_{0}$, $l \geq l_{0}$.

By Proposition 6.1 it holds that for $n \geq n_{0}, l \geq l_{0}$ and any $\delta_{1}>0$,

$$
\begin{aligned}
& \liminf _{\varepsilon \rightarrow 0, \gamma \rightarrow \gamma_{0}} \varepsilon \log P_{\gamma}\left(X_{\varepsilon} \in O\right) \\
& \quad \geq \liminf _{\varepsilon \rightarrow 0, \gamma \rightarrow \gamma_{0}} \varepsilon \log P_{\gamma}\left(X_{\varepsilon} \in U_{n, l}\right) \\
& \quad \geq-\frac{1}{2} \sum_{i=1}^{m_{n}} d\left(\hat{\gamma}^{i}, \gamma_{0}^{i}\right)^{2}\left(1+\delta_{1}\right)-\frac{1}{2}\left(\frac{1}{\delta_{1}}+1\right) \delta_{l}-\sum_{k=m_{n}+1}^{\infty} \frac{1}{2} d\left(\gamma_{0}^{k}, \bar{W}_{r_{n}}^{c}\right)^{2}
\end{aligned}
$$

First letting $l \rightarrow \infty$ and then $\delta_{1} \rightarrow 0$, we obtain that

(6.6) $\liminf _{\varepsilon \rightarrow 0, \gamma \rightarrow \gamma_{0}} \varepsilon \log P_{\gamma}\left(X_{\varepsilon} \in O\right) \geq-\frac{1}{2} \sum_{i=1}^{m_{n}} d\left(\hat{\gamma}^{i}, \gamma_{0}^{i}\right)^{2}-\sum_{k=m_{n}+1}^{\infty} \frac{1}{2} d\left(\gamma_{0}^{k}, \bar{W}_{r_{n}}^{c}\right)^{2}$. 
By the choice of $W_{r_{n}}, d\left(\gamma_{0}^{k}, \bar{W}_{r_{n}}^{c}\right) \leq d\left(\gamma_{0}^{k}, \hat{\gamma}^{k}\right)$ for $k \geq m_{n}+1$. Hence, it follows from (6.6) that

$$
\liminf _{\varepsilon \rightarrow 0, \gamma \rightarrow \gamma_{0}} \varepsilon \ln P_{\gamma_{m}}\left(X_{\varepsilon} \in O\right) \geq-\frac{1}{2} \sum_{i=1}^{\infty} d\left(\hat{\gamma}^{i}, \gamma_{0}^{i}\right)^{2}=-\varrho\left(\gamma_{0}, \hat{\gamma}\right)^{2},
$$

which completes the proof.

Let $\hat{\gamma} \in \Gamma_{\infty}$. To prove Proposition 6.4 we have to consider $d_{\infty}$-neighbourhoods of $\hat{\gamma}$ of the form:

$$
U(\hat{\gamma}, n, \bar{\delta}):=\left\{\gamma=\sum \delta_{\gamma^{i}}|| B_{n}(\gamma)-B_{n}(\hat{\gamma}) \mid<\bar{\delta}\right\} .
$$

and intersections of finitely many of them with vaguely open sets in $\Gamma_{M}$.

Lemma 6.6. Let $\gamma_{0} \in \Gamma_{\infty}$. Then

$$
\liminf _{\varepsilon \rightarrow 0, \gamma \rightarrow \gamma_{0}} \varepsilon \log P_{\gamma}\left(X_{\varepsilon} \in U(\hat{\gamma}, n, \bar{\delta})\right) \geq-\varrho\left(\gamma_{0}, \hat{\gamma}\right)^{2},
$$

where $\gamma \rightarrow \gamma_{0}$ with respect to $d_{\infty}$ in $\Gamma_{\infty}$.

Proof. Let $\left\{\gamma_{m}\right\}$ be a sequence such that $d_{\infty}\left(\gamma_{m}, \gamma_{0}\right) \rightarrow 0$. It suffices to show (6.8) for such a sequence. We may assume $\varrho\left(\gamma_{0}, \hat{\gamma}\right)<\infty$ and

$$
\varrho\left(\gamma_{0}, \hat{\gamma}\right)^{2}=\sum_{i=1}^{\infty} \frac{1}{2} d\left(\gamma_{0}^{i}, \hat{\gamma}^{i}\right)^{2}
$$

where $\gamma_{0}=\sum \delta_{\gamma_{0}^{i}}, \hat{\gamma}=\sum \delta_{\hat{\gamma}^{i}}$. For the numeration of $\gamma_{0}=\sum \delta_{\gamma_{0}^{i}}$, by [KLR03] there exists a numeration of $\gamma_{m}$, say $\gamma_{m}=\sum \delta_{\gamma_{m}^{i}}$, such that for $i \geq 1, \gamma_{m}^{i} \rightarrow \gamma_{0}^{i}$ as $m \rightarrow \infty$. From now on, we stick to such a numeration. For any $N \geq 1$, we have

$$
\begin{aligned}
\sum_{i=N+1}^{\infty} \exp \left[-\frac{1}{2 n} d\left(x_{0}, \gamma_{m}^{i}\right)\right] & =B_{2 n}\left(\gamma_{m}\right)-\sum_{i=1}^{N} \exp \left[-\frac{1}{2 n} d\left(x_{0}, \gamma_{m}^{i}\right)\right] \\
& \rightarrow B_{2 n}\left(\gamma_{0}\right)-\sum_{i=1}^{N} \exp \left[-\frac{1}{2 n} d\left(x_{0}, \gamma_{0}^{i}\right)\right] \\
& =\sum_{i=N+1}^{\infty} \exp \left[-\frac{1}{2 n} d\left(x_{0}, \gamma_{0}^{i}\right)\right]
\end{aligned}
$$

as $m \rightarrow \infty$. 
So we can choose $N_{0}$ so that

$$
\begin{aligned}
& \sum_{i=N_{0}+1}^{\infty} \exp \left[-\frac{1}{2 n} d\left(x_{0}, \hat{\gamma}^{i}\right)\right]<\frac{\bar{\delta}}{4}, \\
& \sum_{i=N_{0}+1}^{\infty} \exp \left[-\frac{1}{2 n} d\left(x_{0}, \gamma_{0}{ }^{i}\right)\right]<\frac{\bar{\delta}}{4}, \\
& \sum_{i=N_{0}+1}^{\infty} \exp \left[-\frac{1}{2 n} d\left(x_{0}, \gamma_{m}{ }^{i}\right)\right]<\frac{\bar{\delta}}{4}
\end{aligned}
$$

for all $m \geq 1$.

Define

$$
\begin{aligned}
U(\hat{\gamma}, n, \bar{\delta}, 1)=\left\{\gamma=\delta_{\gamma^{i}} \mid \exists i_{1}, \ldots, i_{N_{0}}\right. \text { s.t. } & \sum_{j \notin\left\{i_{1}, \ldots, i_{N_{0}}\right\}} \exp \left[-\frac{1}{n} d\left(x_{0}, \gamma^{j}\right)\right]<\frac{\bar{\delta}}{4}, \\
& \left.\left|\sum_{k=1}^{N_{0}} \exp \left[-\frac{1}{n} d\left(x_{0}, \gamma^{i_{k}}\right)\right]-\sum_{k=1}^{N_{0}} \exp \left[-\frac{1}{n} d\left(x_{0}, \hat{\gamma}^{k}\right)\right]\right|<\frac{\bar{\delta}}{4}\right\} .
\end{aligned}
$$

It is easy to see that

$$
U(\hat{\gamma}, n, \bar{\delta}, 1) \subset U(\hat{\gamma}, n, \bar{\delta}) .
$$

Furthermore, there exists $\tilde{\delta}>0$ such that for $\delta_{1} \leq \tilde{\delta}$,

$$
U\left(\hat{\gamma}, n, \bar{\delta}, \delta_{1}\right) \subset U(\hat{\gamma}, n, \bar{\delta}, 1)
$$

where

$$
\begin{aligned}
U\left(\hat{\gamma}, n, \bar{\delta}, \delta_{1}\right)=\left\{\gamma=\delta_{\gamma^{i}} \mid \exists i_{1}, \ldots, i_{N_{0}}\right. \text { s.t. } & \\
& \left.\sum_{j \notin\left\{i_{1}, \ldots, i_{N_{0}}\right\}} \exp \left[-\frac{1}{n} d\left(x_{0}, \gamma^{j}\right)\right]<\frac{\bar{\delta}}{4}, \sum_{k=1}^{N_{0}} d\left(\gamma^{i_{k}}, \hat{\gamma}^{k}\right)^{2}<\delta_{1}\right\} .
\end{aligned}
$$

Now it is enough to prove that

$$
\liminf _{\varepsilon \rightarrow 0, m \rightarrow \infty} \varepsilon \log P_{\gamma_{m}}\left(X_{\varepsilon} \in U(\hat{\gamma}, n, \bar{\delta}, 1)\right) \geq-\varrho\left(\gamma_{0}, \hat{\gamma}\right)^{2} .
$$


For $\delta_{1} \leq \tilde{\delta}$, we have

$$
\begin{aligned}
P_{\gamma_{m}} & \left.X_{\varepsilon} \in U(\hat{\gamma}, n, \bar{\delta}, 1)\right) \\
& \geq P_{\gamma_{m}}\left(X_{\varepsilon} \in U\left(\hat{\gamma}, n, \bar{\delta}, \delta_{1}\right)\right) \\
& \geq P_{\gamma_{m}}\left(\sum_{i=N_{0}+1}^{\infty} \exp \left[-\frac{1}{n} d\left(x_{0}, B_{\varepsilon}{ }^{i}\right)\right]<\frac{\bar{\delta}}{4}, \sum_{i=1}^{N_{0}} d\left(B_{\varepsilon}{ }^{i}, \hat{\gamma}^{i}\right)^{2}<\delta_{1}\right)
\end{aligned}
$$

(where $B_{\varepsilon}{ }^{i}$ is the Brownian motion starting at $\gamma_{m}^{i}$ )

$$
\begin{aligned}
= & P_{\gamma_{m}}\left(\sum_{i=1}^{N_{0}} d\left(B_{\varepsilon}{ }^{i}, \hat{\gamma}^{i}\right)^{2}<\delta_{1}\right) \\
& \times P_{\gamma_{m}}\left(\sum_{i=N_{0}+1}^{\infty} \exp \left[-\frac{1}{n} d\left(x_{0}, B_{\varepsilon}{ }^{i}\right)\right]<\frac{\bar{\delta}}{4}\right) \\
:= & a_{\varepsilon}^{m} \times b_{\varepsilon}^{m} .
\end{aligned}
$$

Note that for any $\delta_{2}>0$,

$$
\sum_{i=1}^{N_{0}} d\left(x^{i}, \gamma_{m}^{i}\right)^{2} \leq\left(1+\frac{1}{\delta_{2}}\right) \sum_{i=1}^{N_{0}} d\left(x^{i}, \hat{\gamma}^{i}\right)^{2}+\left(1+\delta_{2}\right) \sum_{i=1}^{N_{0}} d\left(\hat{\gamma}^{i}, \gamma_{m}^{i}\right)^{2} .
$$

Using

$$
a_{\varepsilon}^{m}=\int_{\sum_{i=1}^{N_{0}} d\left(x^{i}, \hat{\gamma}^{i}\right)^{2}<\delta_{1}} \prod_{i=1}^{N_{0}} p_{\varepsilon}\left(\gamma_{m}^{i}, x^{i}\right) m\left(d x^{1}\right) \ldots m\left(d x^{N_{0}}\right)
$$

as in the proof of Proposition 6.1, we have

(6.10) $\liminf _{\varepsilon \rightarrow 0, m \rightarrow \infty} \varepsilon \log a_{\varepsilon}^{m} \geq-\frac{1}{2-\delta}\left(1+\frac{1}{\delta_{2}}\right) \delta_{1}-\frac{1}{2-\delta}\left(1+\delta_{2}\right) \sum_{i=1}^{N_{0}} d\left(\gamma_{0}^{i}, \hat{\gamma}^{i}\right)^{2}$.

Next we are going to show that

$$
\liminf _{\varepsilon \rightarrow 0, m \rightarrow \infty} \varepsilon \log b_{\varepsilon}^{m}=0 .
$$

For this end, it is sufficient to establish

$$
\liminf _{\varepsilon \rightarrow 0, m \rightarrow \infty} b_{\varepsilon}^{m}>0
$$


By the choice of $N_{0}$, it is easy to see that

$$
\begin{aligned}
\left\{\sum_{i=N_{0}+1}^{\infty} \exp \left[-\frac{1}{n} d\left(x_{0}, B_{\varepsilon}{ }^{i}\right)\right]<\frac{\bar{\delta}}{4}\right\} & \supset \bigcap_{i=N_{0}+1}^{\infty}\left\{d\left(x_{0}, B_{\varepsilon}{ }^{i}\right)>\frac{1}{2} d\left(x_{0}, \gamma_{m}^{i}\right)\right\} \\
& \supset \bigcap_{i=N_{0}+1}^{\infty}\left\{d\left(\gamma_{m}^{i}, B_{\varepsilon}{ }^{i}\right)<\frac{1}{2} d\left(x_{0}, \gamma_{m}^{i}\right)\right\}
\end{aligned}
$$

since $d\left(x_{0}, \gamma_{m}^{i}\right) \leq d\left(x_{0}, B_{\varepsilon}{ }^{i}\right)+d\left(\gamma_{m}^{i}, B_{\varepsilon}{ }^{i}\right)$.

Hence, by Lemma 3.1, for $\varepsilon \leq 1$,

$$
\begin{aligned}
b_{\varepsilon}^{m} & =P_{\gamma_{m}}\left(\sum_{i=N_{0}+1}^{\infty} \exp \left[-\frac{1}{n} d\left(x_{0}, B_{\varepsilon}{ }^{i}\right)\right]<\frac{\bar{\delta}}{4}\right) \\
& \geq \prod_{i=N_{0}+1}^{\infty} P_{\gamma_{m}^{i}}\left(d\left(\gamma_{m}^{i}, B_{\varepsilon}{ }^{i}\right)<\frac{1}{2} d\left(x_{0}, \gamma_{m}^{i}\right)\right) \\
& \geq \prod_{i=N_{0}+1}^{\infty}\left(1-c_{1} \exp \left(-\frac{c_{2} d\left(x_{0}, \gamma_{m}^{i}\right)^{2}}{4 \varepsilon}\right)\right) \\
& \geq \prod_{i=N_{0}+1}^{\infty}\left(1-c_{1} \exp \left(-\frac{c_{2} d\left(x_{0}, \gamma_{m}^{i}\right)^{2}}{4}\right)\right) \\
& =\exp \left[\sum_{i=N_{0}+1}^{\infty} \log \left(1-c_{1} \exp \left(-\frac{c_{2} d\left(x_{0}, \gamma_{m}^{i}\right)^{2}}{4}\right)\right)\right] \\
& \geq \exp \left[-\frac{1}{2} \sum_{i=1}^{\infty} c_{1} \exp \left(-\frac{c_{2} d\left(x_{0}, \gamma_{m}^{i}\right)^{2}}{4}\right)\right] .
\end{aligned}
$$

The last expression is bigger than some positive constant independent of $m$ since $\sup _{m} B_{n}\left(\gamma_{m}\right)<\infty$ for all $n \geq 1$. Therefore, (6.11) follows.

Combining (6.10) and (6.11) we arrive at

$$
\begin{aligned}
\liminf _{\varepsilon \rightarrow 0, \gamma \rightarrow \gamma_{0}} \varepsilon \log P_{\gamma}\left(X_{\varepsilon} \in U(\hat{\gamma}, n, \bar{\delta})\right) \geq & -\frac{1}{2-\delta}\left(1+\frac{1}{\delta_{2}}\right) \delta_{1} \\
& -\frac{1}{2-\delta}\left(1+\delta_{2}\right) \sum_{i=1}^{N_{0}} d\left(\gamma_{0}^{i}, \hat{\gamma}^{i}\right)^{2} .
\end{aligned}
$$

Letting first $\delta_{1} \rightarrow 0$, then $\delta_{2} \rightarrow 0$ and finally $\delta \rightarrow 0$ we get

$$
\liminf _{\varepsilon \rightarrow 0, \gamma \rightarrow \gamma_{0}} \varepsilon \log P_{\gamma}\left(X_{\varepsilon} \in U(\hat{\gamma}, n, \bar{\delta})\right) \geq-\frac{1}{2} \sum_{i=1}^{N_{0}} d\left(\gamma_{0}^{i}, \hat{\gamma}^{i}\right)^{2} \geq-\varrho\left(\gamma_{0}, \hat{\gamma}\right)^{2} .
$$

This completes the proof. 
We will sketch the case for the intersection of two $d_{\infty}$-neighbourhoods in the next lemma.

Let $z_{0} \in \mathbf{R}, \bar{\delta}>0$. Define

$$
U\left(z_{0}, n, \bar{\delta}\right):=\left\{\gamma=\sum \delta_{\gamma^{i}}|| B_{n}(\gamma)-z_{0} \mid<\bar{\delta}\right\} .
$$

Let $U$ be an $d_{v}$-open neighborhood in $\Gamma_{M}$ described by

$$
U=\left\{\gamma \in \Gamma_{M}\left|\gamma\left(\partial W_{r_{0}}\right)=0, \gamma\right|_{W_{r_{0}}}=\sum_{i=1}^{n_{0}} \delta_{x_{i}} \text { with } \sum_{i=1}^{n_{0}} d\left(x_{i}, y_{i}\right)^{2}<\delta_{0}\right\},
$$

where $W_{r_{0}}:=B\left(x_{0}, r_{0}\right), r_{0}>0, y_{1}, \ldots, y_{n_{0}}$ are fixed points in $W_{r_{0}}$, and $\delta_{0}>0$. Set $V=U\left(z_{0}, n, \bar{\delta}\right) \cap U$.

Lemma 6.7. Let $\gamma_{0} \in \Gamma_{\infty}$. Then for any $\hat{\gamma} \in V$, we have

$$
\liminf _{\varepsilon \rightarrow 0, \gamma \rightarrow \gamma_{0}} \varepsilon \log P_{\gamma}\left(X_{\varepsilon} \in V\right) \geq-\varrho\left(\gamma_{0}, \hat{\gamma}\right)^{2},
$$

where $\gamma \rightarrow \gamma_{0}$ with respect to $d_{\infty}$ in $\Gamma_{\infty}$.

Proof. Let $\left\{\gamma_{m}\right\}$ be a sequence such that $d_{\infty}\left(\gamma_{m}, \gamma_{0}\right) \rightarrow 0$. It suffices to show (6.14) for such a sequence. Again, we may assume $\varrho\left(\gamma_{0}, \hat{\gamma}\right)<\infty$ and

$$
\varrho\left(\gamma_{0}, \hat{\gamma}\right)^{2}=\sum_{i=1}^{\infty} \frac{1}{2} d\left(\gamma_{0}^{i}, \hat{\gamma}^{i}\right)^{2}
$$

where $\gamma_{0}=\sum \delta_{\gamma_{0}^{i}}, \hat{\gamma}=\sum \delta_{\hat{\gamma}^{i}}$. For the numeration of $\gamma_{0}=\sum \delta_{\gamma_{0}^{i}}$, by [KLR03] there exists a numeration of $\gamma_{m}$, say $\gamma_{m}=\sum \delta_{\gamma_{m}^{i}}$, such that for $i \geq 1, \gamma_{m}^{i} \rightarrow \gamma_{0}^{i}$ as $m \rightarrow \infty$. We will stick to such a numeration. Let $\delta_{1}:=\bar{\delta}-\left|B_{n}(\hat{\gamma})-z_{0}\right|$. Since $\hat{\gamma} \in U\left(z_{0}, n, \bar{\delta}\right), \delta_{1}>0$. Choose $\delta_{2}<\frac{\delta_{1}}{4}$ such that $\exp \left(-\frac{1}{2 n} d\left(x_{0}, x\right)\right)<\delta_{2}$ implies $d\left(x_{0}, x\right)>r_{0}$, where $r_{0}$ is as in the definition of $U$. On the other hand, since $\hat{\gamma} \in U$, there exist $\hat{\gamma}^{i_{k}} \in \hat{\gamma}, k=1, \ldots, n_{0}$, such that

$$
\left.\hat{\gamma}\right|_{W_{r_{0}}}=\sum_{k=1}^{n_{0}} \delta_{\hat{\gamma}^{i k}}, \sum_{k=1}^{n_{0}} d\left(\hat{\gamma}^{i_{k}}, y_{k}\right)^{2}<\delta_{0}
$$

Now, arguing as in the proof of Proposition 6.6, we can choose $N_{0} \geq i_{n_{0}}$ so 
that

$$
\begin{array}{r}
\sum_{i=N_{0}+1}^{\infty} \exp \left[-\frac{1}{2 n} d\left(x_{0}, \hat{\gamma}^{i}\right)\right]<\frac{\bar{\delta}}{4}, \\
\sum_{i=N_{0}+1}^{\infty} \exp \left[-\frac{1}{2 n} d\left(x_{0}, \gamma_{0}{ }^{i}\right)\right]<\frac{\bar{\delta}}{4}, \\
\sum_{i=N_{0}+1}^{\infty} \exp \left[-\frac{1}{2 n} d\left(x_{0}, \gamma_{m}{ }^{i}\right)\right]<\frac{\bar{\delta}}{4},
\end{array}
$$

for all $m \geq 1$. For such $N_{0}$, there exists $\tilde{\delta}>0$ such that for all $\delta_{3} \leq \tilde{\delta}$ and $\left(z_{1}, z_{2}, \ldots, z_{N_{0}}\right) \in M^{N_{0}},\left|\sum_{i=1}^{N_{0}} d\left(z_{i}, \hat{\gamma}^{i}\right)^{2}\right|<\delta_{3}$ implies

(i) $\mid \sum_{i=1}^{N_{0}} \exp \left(-\frac{1}{2 n} d\left(x_{0}, z_{i}\right)-\sum_{i=1}^{N_{0}} \exp \left(-\frac{1}{2 n} d\left(x_{0}, \hat{\gamma}^{i}\right) \mid<\delta_{1}\right.\right.$,

(ii) $\sum_{k=1}^{n_{0}} d\left(z_{i_{k}}, y_{k}\right)^{2}<\delta_{0}$,

(iii) $\sharp\left\{i ; z_{i} \in W_{r_{0}}\right\}=n_{0}$.

So, for $\delta_{3} \leq \tilde{\delta}$,

$$
U\left(\hat{\gamma}, n, \delta_{1}, \delta_{3}\right) \subset V
$$

where

$$
\begin{aligned}
U\left(\hat{\gamma}, n, \delta_{1}, \delta_{3}\right)= & \left\{\gamma=\sum_{i} \delta_{\gamma^{i}} \mid \exists i_{1}, \ldots, i_{N_{0}}\right. \text { s.t. } \\
& \left.\sum_{j \notin\left\{i_{1}, \ldots, i_{N_{0}}\right\}} \exp \left[-\frac{1}{2 n} d\left(x_{0}, \gamma^{j}\right)\right]<\frac{\bar{\delta}}{4}, \sum_{k=1}^{N_{0}} d\left(\gamma^{i_{k}}, \hat{\gamma}^{k}\right)^{2}<\delta_{1}\right\} .
\end{aligned}
$$

Now it is enough to prove that

$$
\liminf _{\varepsilon \rightarrow 0, m \rightarrow \infty} \varepsilon \log P_{\gamma_{m}}\left(X_{\varepsilon} \in U\left(\hat{\gamma}, n, \delta_{1}, \delta_{3}\right)\right) \geq-\varrho\left(\gamma_{0}, \hat{\gamma}\right)^{2} .
$$

The rest of the proof is exactly the same as the corresponding proof of Proposition 6.6. So, we omit it here.

Finite intersections can be done similarly. Since arbitrary finite intersections of $d_{\infty}$-neighbourhoods as above form a base for the $d_{\infty}$-neighbourhoods of any $\hat{\gamma} \in \Gamma_{\infty}$, we can then prove Proposition 6.4 in the same way as Proposition 6.5 .

Theorem 6.8. Let $\gamma_{0} \in \Gamma_{\infty}$ and let $\mu_{\varepsilon}$ be the law of $Y^{\varepsilon}$ on $\Gamma_{M}^{n}$ under $P_{\gamma_{0}}$. Then for any subset $G \subset \Gamma_{M}^{n}$, which is open with respect to the product topology induced by $d_{v}$,

$$
\liminf _{\varepsilon \rightarrow 0} \varepsilon \log \mu_{\varepsilon}(G) \geq-\inf _{\left(\gamma_{1}, \ldots, \gamma_{n}\right) \in G} I_{\gamma_{0}}\left(\gamma_{1}, \ldots, \gamma_{n}\right)
$$


Proof. It suffices to show that for any $\left(\gamma_{1}, \gamma_{2}, \ldots, \gamma_{n}\right) \in G$ with $I_{\gamma_{0}}\left(\gamma_{1}, \ldots\right.$, $\left.\gamma_{n}\right)<\infty$

$$
\liminf _{\varepsilon \rightarrow 0} \varepsilon \log \mu_{\varepsilon}(G) \geq-I_{\gamma_{0}}\left(\gamma_{1}, \ldots, \gamma_{n}\right) .
$$

Obviously, if $\gamma, \tilde{\gamma} \in \Gamma_{M}$ such that $\varrho(\gamma, \tilde{\gamma})<\infty$ and $\gamma \in \Gamma_{\infty}$, then $\tilde{\gamma} \in \Gamma_{\infty}$. So, we may assume that $\gamma_{1}, \ldots, \gamma_{n} \in \Gamma_{\infty}$, since so is $\gamma_{0}$. For $\gamma \in \Gamma_{\infty}$, let $P_{t}(\gamma, U):=$ $P_{\gamma}\left(X_{t} \in U\right)$ be the transition function. For a $d_{\infty}$-open neighbourhood $O_{n}$ of $\gamma_{n}$ in $\Gamma_{\infty}$, it follows from Proposition 6.4 that

$$
\liminf _{\varepsilon \rightarrow 0, \gamma \rightarrow \gamma_{n-1}} \varepsilon \log P_{\varepsilon\left(t_{n}-t_{n-1}\right)}\left(\gamma, O_{n}\right) \geq-\frac{\varrho\left(\gamma_{n-1}, \gamma_{n}\right)^{2}}{t_{n}-t_{n-1}},
$$

where $\gamma \rightarrow \gamma_{n-1}$ with respect to $d_{\infty}$ in $\Gamma_{\infty}$. Thus, given $\delta_{n}>0$, there exist $\varepsilon_{n}>0$ and a $d_{\infty}$-open neighbourhood $O_{n-1}$ of $\gamma_{n-1}$ in $\Gamma_{\infty}$ such that

$$
P_{\varepsilon\left(t_{n}-t_{n-1}\right)}\left(\gamma, O_{n}\right) \geq \exp \left[-\frac{1}{\varepsilon}\left(\frac{\varrho\left(\gamma_{n-1}, \gamma_{n}\right)^{2}}{t_{n}-t_{n-1}}-\delta_{n}\right)\right] \text { for } \varepsilon \leq \varepsilon_{n}, \gamma \in O_{n-1} .
$$

The same arguments imply that for $\delta_{1}>0, \delta_{2}>0, \ldots, \delta_{n-1}>0$ there exist $d_{\infty}$-open neighbourhoods $O_{i}$ of $\gamma_{i}$ in $\Gamma_{\infty}$ and $\varepsilon_{i}>0, i=1,2, \ldots, n-1$, such that

$$
P_{\varepsilon\left(t_{i}-t_{i-1}\right)}\left(\gamma, O_{i}\right) \geq \exp \left[-\frac{1}{\varepsilon}\left(\frac{\varrho\left(\gamma_{i-1}, \gamma_{i}\right)^{2}}{t_{i}-t_{i-1}}-\delta_{i}\right)\right] \text { for } \varepsilon \leq \varepsilon_{i}, \gamma \in O_{i-1} .
$$

Moreover, making them smaller if necessary, we may assume $O_{1} \times O_{2} \times \cdots \times O_{n} \subset$ $G$. Then for $\varepsilon \leq \varepsilon_{1} \wedge \varepsilon_{2} \wedge \cdots \wedge \varepsilon_{n}$, we have

$$
\begin{aligned}
\mu_{\varepsilon}(G) \geq & \mu_{\varepsilon}\left(O_{1} \times O_{2} \times \cdots \times O_{n}\right) \\
= & \int_{O_{1}} P_{\varepsilon t_{1}}\left(\gamma_{0}, d \eta_{1}\right)\left(\int_{O_{2}} P_{\varepsilon\left(t_{2}-t_{1}\right)}\left(\eta_{1}, d \eta_{2}\right)\right) \\
& \times \cdots \times \int_{O_{n-1}} P_{\varepsilon\left(t_{n-1}-t_{n-2}\right)}\left(\eta_{n-2}, d \eta_{n-1}\right) \times P_{\varepsilon\left(t_{n}-t_{n-1}\right)}\left(\eta_{n-1}, O_{n}\right) \\
\geq & \exp \left[-\frac{1}{\varepsilon}\left(\frac{\varrho\left(\gamma_{n-1}, \gamma_{n}\right)^{2}}{t_{n}-t_{n-1}}-\delta_{n}\right)\right] \int_{O_{1}} P_{\varepsilon t_{1}}\left(\gamma_{0}, d \eta_{1}\right) \\
& \int_{O_{2}} P_{\varepsilon\left(t_{2}-t_{1}\right)}\left(\eta_{1}, d \eta_{2}\right) \times \cdots \times \int_{O_{n-2}} P_{\varepsilon\left(t_{n-2}-t_{n-3}\right)}\left(\eta_{n-3}, d \eta_{n-2}\right) \\
& P_{\varepsilon\left(t_{n-1}-t_{n-2}\right)}\left(\eta_{n-2}, O_{n-1}\right) \\
\geq & \cdots \geq \exp \left[-\frac{1}{\varepsilon}\left(\sum_{k=1}^{n} \frac{\varrho\left(\gamma_{k-1}, \gamma_{k}\right)^{2}}{t_{k}-t_{k-1}}+\sum_{k=1}^{n} \delta_{k}\right)\right] .
\end{aligned}
$$


Therefore,

$$
\liminf _{\varepsilon \rightarrow 0} \varepsilon \log \mu_{\varepsilon}(G) \geq-I_{\gamma_{0}}\left(\gamma_{1}, \ldots, \gamma_{n}\right)-\sum_{k=1}^{n} \delta_{k} .
$$

Letting $\sum_{k=1}^{n} \delta_{k} \rightarrow 0$, the theorem follows.

\section{$\S 7 . \quad$ The Sample Path Large Deviations}

In this section we fix $\gamma_{0} \in \Gamma_{\infty}$. Let $P^{\varepsilon}$ denote the law of $\left(X_{\varepsilon t}\right)_{t \in[0,1]}$ on $C_{\gamma_{0}}\left([0,1] \rightarrow \Gamma_{M}\right)$, i.e., the set of all continuous paths $t \rightarrow \omega_{t}$ from $[0,1]$ to $\Gamma_{M}$ such that $\omega_{0}=\gamma_{0}$. We equip $C_{\gamma_{0}}\left([0,1] \rightarrow \Gamma_{M}\right)$ with the topology of uniform convergence, where $\Gamma_{M}$ is equipped with the vague topology. For $\omega \in C_{\gamma_{0}}\left([0,1] \rightarrow \Gamma_{M}\right)$, define

$$
I(\omega):=\sup _{0=t_{0}<t_{1}<\cdots<t_{n}=1}\left\{\sum_{k=1}^{n} \frac{\varrho\left(\omega_{t_{k-1}}, \omega_{t_{k}}\right)^{2}}{t_{k}-t_{k-1}}\right\},
$$

where the supremum is taken over all finite partitions of the interval $[0,1] . I(\omega)$ is the energy of $\omega$ associated to the Wasserstein type distance $\varrho$. The function $I$ is obviously lower semicontinuous. Furthermore, by [RS99, Lemma 4.1(vii)] closed $\varrho$-balls are compact. Therefore, since for $\omega \in\{I \leq$ const. $\}$ we have $\omega([0,1]) \subset\left\{\varrho\left(\gamma_{0}, \cdot\right)<\infty\right\}$ and since on $\left\{\varrho\left(\gamma_{0}, \cdot\right)<\infty\right\}$ the $\varrho$-topology is stronger than the $d_{v}$-topology, Arzela's Theorem implies that $\{I \leq$ const. $\}$ is compact in $C_{\gamma_{0}}\left([0,1] \rightarrow \Gamma_{M}\right)$.

Theorem 7.1. $\quad\left\{P^{\varepsilon}, \varepsilon>0\right\}$ satisfies a large deviation principle on $C_{\gamma_{0}}$ $\left([0,1] \rightarrow \Gamma_{M}\right)$ with good rate function $I(\cdot)$ given in $(7.1)$, i.e.,

(i) for any closed subset $C \subset C_{\gamma_{0}}\left([0,1] \rightarrow \Gamma_{M}\right)$,

$$
\limsup _{\varepsilon \rightarrow 0} \varepsilon \log P^{\varepsilon}(C) \leq-\inf _{\omega \in C} I(\omega) .
$$

(ii) for any open subset $O \subset C_{\gamma_{0}}\left([0,1] \rightarrow \Gamma_{M}\right)$,

$$
\liminf _{\varepsilon \rightarrow 0} \varepsilon \log P^{\varepsilon}(O) \geq-\inf _{\omega \in O} I(\omega) .
$$

Proof. The results in Sections 4 and 6 imply (see Theorem 4.6.1 in [DZ92]) that $\left\{P^{\varepsilon}, \varepsilon>0\right\}$ satisfies a large deviation principle under a weaker topology on $C_{\gamma_{0}}\left([0,1] \rightarrow \Gamma_{M}\right)$ with the same good rate function $I(\cdot)$. So, the assertion follows from the exponential tightness proven in Proposition 7.3 below.

We need the following lemma. 
Lemma 7.2. Let $\hat{B}$ denote a real valued standard Brownian motion. Let $c_{0}$ be a constant satisfying

$$
A:=E\left[\exp \left(c_{0} \sup _{0 \leq u \leq 1} \hat{B}_{u}^{2}\right)\right]<\infty .
$$

For $f \in C_{0}^{2}(M)$, set $\lambda_{f}=\frac{c_{0}}{2\|\nabla f\|_{\infty}^{2}+1}$. Then for $0 \leq s<t \leq 1$,

$$
E\left[\exp \left(\lambda_{f} \frac{\left(f\left(B_{t}\right)-f\left(B_{s}\right)\right)^{2}}{t-s}\right)\right] \leq A \exp \left(\|\Delta f\|_{\infty}^{2}\right) .
$$

Proof. By Ito's formula,

$$
f\left(B_{t}\right)-f\left(B_{s}\right)=M_{t}^{f}-M_{s}^{f}+\int_{s}^{t} \frac{1}{2} \Delta f\left(B_{u}\right) d u,
$$

where $M^{f}$ is a martingale with $\left\langle M^{f}\right\rangle_{t}=\int_{0}^{t}|\nabla f|^{2}\left(B_{u}\right) d u$. This implies

$$
\frac{\left(f\left(B_{t}\right)-f\left(B_{s}\right)\right)^{2}}{t-s} \leq 2 \frac{\left(M_{t}^{f}-M_{s}^{f}\right)^{2}}{t-s}+\|\Delta f\|_{\infty}^{2} .
$$

By the martingale representation theorem, there is a real-valued Brownian motion $\hat{B}$ such that $M_{t}^{f}-M_{s}^{f}=\hat{B}_{\left\langle M^{f}\right\rangle_{t}-\left\langle M^{f}\right\rangle_{s}}$. Thus, it follows from (7.4) that

$$
\begin{aligned}
\frac{\left(f\left(B_{t}\right)-f\left(B_{s}\right)\right)^{2}}{t-s} & \leq 2 \frac{\left(\hat{B}_{\int_{s}^{t}|\nabla f|^{2}\left(B_{u}\right) d u}\right)^{2}}{t-s}+\|\Delta f\|_{\infty}^{2} \\
& \leq 2 \sup _{0 \leq u \leq\|\nabla f\|_{\infty}^{2}(t-s)} \frac{\hat{B}_{u}^{2}}{t-s}+\|\Delta f\|_{\infty}^{2} \\
& =2\|\nabla f\|_{\infty}^{2} \sup _{0 \leq u \leq 1} \tilde{B}_{u}^{2}+\|\Delta f\|_{\infty}^{2},
\end{aligned}
$$

where $\tilde{B}_{u}:=\frac{1}{\sqrt{t-s}} \hat{B}_{(t-s) u}$ is again a real-valued Brownian motion and we used the scaling invariance property of standard Brownian motion in the last step. Hence,

$$
\begin{aligned}
& E\left[\exp \left(\lambda_{f} \frac{\left(f\left(\hat{B}_{t}\right)-f\left(\hat{B}_{s}\right)\right)^{2}}{t-s}\right)\right] \\
& \leq E\left[\exp \left(\lambda_{f} 2\|\nabla f\|_{\infty}^{2} \sup _{0 \leq u \leq 1} \tilde{B}_{u}^{2}+\|\Delta f\|_{\infty}^{2}\right)\right] \\
& \leq A \exp \left(\|\Delta f\|_{\infty}^{2}\right) .
\end{aligned}
$$

Let $P^{\varepsilon}$ be the law of $\left(X_{\varepsilon t}\right)_{t \in[0,1]}$ on $C_{\gamma_{0}}\left([0,1] \rightarrow \Gamma_{M}\right)$. 
Proposition 7.3. $\left\{P^{\varepsilon}, \varepsilon>0\right\}$ is exponentially tight on $C\left([0,1] \rightarrow \Gamma_{M}\right)$, i.e., for any $L>0$, there exists a compact subset $K_{L} \subset C_{\gamma_{0}}\left([0,1] \rightarrow \Gamma_{M}\right)$ satisfying

$$
\limsup _{\varepsilon \rightarrow \infty} \log P^{\varepsilon}\left(K_{L}^{c}\right) \leq-L
$$

Proof. Fix a countable, dense subset $\left\{f_{n}, n \geq 1\right\}$ of $C_{0}(M)$ such that $f_{n} \in C_{0}^{2}(M)$ and the family $\left\{f_{n}, n \geq 1\right\}$ is closed under addition. For $L_{n}>0$, $n \in \mathbf{N}$, define a subset of $C_{\gamma_{0}}([0,1] \rightarrow \chi)$ by

$$
\begin{aligned}
K_{\left\{L_{n}\right\}}:=\bigcap_{n=1}^{\infty}\left\{\omega \in C_{\gamma_{0}}\left([0,1] \rightarrow \Gamma_{M}\right) \mid\right. \\
\left.\quad\left|\left\langle\omega_{t}, f_{n}\right\rangle-\left\langle w_{s}, f_{n}\right\rangle\right| \leq L_{n}|t-s|^{\frac{1}{2}-\delta}, 0 \leq s, t \leq 1\right\}
\end{aligned}
$$

where $0<\delta<\frac{1}{2}$ is fixed. It is known from [Kal97] that $K$ is compact. For $L>0$, we will choose $L_{n}$ properly so that (7.8) is satisfied with $K_{L}:=K_{\left\{L_{n}\right\}}$. Suppose $\operatorname{supp}\left[f_{n}\right] \subset B\left(x_{0}, N_{n}\right)$. Select an integer $k_{n}$ such that

$$
d\left(x_{0}, \gamma_{0}^{j}\right) \geq\left(N_{n}+1 \sqrt{2(n+L)}\right) \vee\left(2 N_{n}+1\right)
$$

for all $j \geq k_{n}$. This is possible as $\lim _{j \rightarrow \infty} d\left(x_{0}, \gamma_{0}^{j}\right)=+\infty$. Set

$$
c_{\delta}:=\sup _{0 \leq u \leq 1}\left|\log \left(u^{2}\right) u^{\delta}\right|, \lambda_{n}:=\frac{c_{0}}{\left(2\left\|\nabla f_{n}\right\|_{\infty}^{2}+1\right) k_{n}} .
$$

where $c_{0}$ is as in Lemma 7.2. Now, choose $L_{n}$ such that

$$
\left(\frac{\sqrt{\lambda_{n}} L_{n}}{8 c_{\delta}}-1\right)^{2} \geq L+n+k_{n}\left\|\Delta f_{n}\right\|_{\infty}^{2}+k_{n} \log (A),
$$

where $A$ is as in Lemma 7.1. Define $K_{L}:=K_{\left\{L_{n}\right\}}$ with $K_{\left\{L_{n}\right\}}$ as in (7.9). We claim that $K_{L}$ satisfies (7.8). To prove this we set

$$
\begin{aligned}
A_{n}:=\left\{\omega \in C_{\gamma_{0}}([0,1]\right. & \left.\rightarrow \Gamma_{M}\right) \mid \\
& \left.\left|\left\langle\omega_{t}, f_{n}\right\rangle-\left\langle w_{s}, f_{n}\right\rangle\right| \leq L_{n}|t-s|^{\frac{1}{2}-\delta}, 0 \leq s, t \leq 1\right\} .
\end{aligned}
$$

Then

$$
P^{\varepsilon}\left(K_{L}^{c}\right) \leq \sum_{n=1}^{\infty} P^{\varepsilon}\left(A_{n}^{c}\right)
$$


Since the support of $f_{n}$ is contained in $B\left(x_{0}, N_{n}\right)$, we have

$$
\begin{aligned}
& P^{\varepsilon}\left(A_{n}^{c}\right)= P_{\gamma_{0}}\left(A_{n}^{c} \bigcap\left\{\inf _{j \geq k_{n}} \inf _{u \leq 1} d\left(x_{0}, B_{\varepsilon u}^{j}\right) \leq N_{n}\right\}\right) \\
&+P_{\gamma_{0}}\left(A_{n}^{c} \bigcap\left\{\inf _{j \geq k_{n}} \inf _{u \leq 1} d\left(x_{0}, B_{\varepsilon u}^{j}\right)>N_{n}\right\}\right) \\
& \leq P_{\gamma_{0}}\left(\inf _{j \geq k_{n}} \inf _{u \leq 1} d\left(x_{0}, B_{\varepsilon u}^{j}\right) \leq N_{n}\right) \\
&+P_{\gamma_{0}}\left(\left|\sum_{j=1}^{k_{n}} f_{n}\left(B_{\varepsilon t}^{j}\right)-f_{n}\left(B_{\varepsilon s}^{j}\right)\right|>L_{n}|t-s|^{\frac{1}{2}-\delta}\right. \\
&\quad \text { for some } 0 \leq s \leq t \leq 1) \\
&=: I_{n}^{\varepsilon}+I I_{n}^{\varepsilon} .
\end{aligned}
$$

Clearly,

$$
\begin{aligned}
I_{n}^{\varepsilon} & \leq \sum_{j \geq k_{n}}^{\infty} P_{\gamma_{0}}\left(\inf _{u \leq 1} d\left(x_{0}, B_{\varepsilon u}^{j}\right)<N_{n}+1\right) \\
& =\sum_{j \geq k_{n}}^{\infty} P_{\gamma_{0}}\left(\inf _{u \leq 1} d\left(x_{0}, B_{\varepsilon u}^{j}\right) \leq N_{n}+1\right. \\
& \left.\sup _{u \leq 1} d\left(\gamma_{0}^{j}, B_{\varepsilon u}^{j}\right) \geq d\left(x_{0}, \gamma_{0}^{j}\right)-\inf _{u \leq 1} d\left(x_{0}, B_{\varepsilon u}^{j}\right)\right) \\
& \leq \sum_{j \geq k_{n}}^{\infty} P_{\gamma_{0}}\left(\sup _{u \leq 1} d\left(\gamma_{0}^{j}, B_{\varepsilon u}^{j}\right) \geq d\left(x_{0}, \gamma_{0}^{j}\right)-N_{n}-1\right) .
\end{aligned}
$$

By Corollary 3.2 and setting $c_{1}:=c_{1}(1), c_{2}:=c_{2}(1)$,

$$
\begin{aligned}
P_{\gamma_{0}}\left(\sup _{u \leq 1} d\left(\gamma_{0}^{j}, B_{\varepsilon u}^{j}\right) \geq d\left(x_{0}, \gamma_{0}^{j}\right)\right. & \left.-N_{n}-1\right) \\
\leq & c_{1} \exp \left(-c_{2} \frac{\left(d\left(x_{0}, \gamma_{0}^{j}\right)-N_{n}-1\right)^{2}}{\varepsilon}\right)
\end{aligned}
$$

Combining (7.13), (7.14) and the choice of $k_{n}$ we arrive at

$$
I_{n}^{\varepsilon} \leq \sum_{j \geq k_{n}}^{\infty} c_{1} \exp \left(-c_{2} \frac{\left(d\left(x_{0}, \gamma_{0}^{j}\right)-N_{n}-1\right)^{2}}{\varepsilon}\right)
$$




$$
\begin{aligned}
& =\sum_{j \geq k_{n}}^{\infty} c_{1} \exp \left(-\frac{(n+L)}{\varepsilon}\right) \exp \left(-c_{2} \frac{1}{2} \frac{\left(d\left(x_{0}, \gamma_{0}^{j}\right)-N_{n}-1\right)^{2}}{\varepsilon}\right) \\
& \leq c_{1} \exp \left(-\frac{(n+L)}{\varepsilon}\right) \sum_{j \geq k_{n}}^{\infty} \exp \left(-c_{2} \frac{1}{8} \frac{d\left(x_{0}, \gamma_{0}^{j}\right)^{2}}{\varepsilon}\right) \\
& \leq \hat{c}_{1} \exp \left(-\frac{(n+L)}{\varepsilon}\right)
\end{aligned}
$$

since $\gamma_{0} \in \Gamma_{\infty}$. For the term $I I_{n}^{\varepsilon}$, we note that by Lemma 7.2,

$$
\begin{aligned}
& E\left[\exp \left(\lambda_{n} \frac{\left|\sum_{j=1}^{k_{n}} f_{n}\left(B_{\varepsilon t}^{j}\right)-f_{n}\left(B_{\varepsilon s}^{j}\right)\right|^{2}}{\varepsilon(t-s)}\right)\right] \\
\leq & E\left[\exp \left(\lambda_{n} k_{n} \sum_{j=1}^{k_{n}} \frac{\left(f_{n}\left(B_{\varepsilon t}^{j}\right)-f_{n}\left(B_{\varepsilon s}^{j}\right)\right)^{2}}{\varepsilon(t-s)}\right)\right] \\
= & \prod_{j=1}^{k_{n}} E\left[\exp \left(\lambda_{n} k_{n} \frac{\left(f_{n}\left(B_{\varepsilon t}^{j}\right)-f_{n}\left(B_{\varepsilon s}^{j}\right)\right)^{2}}{\varepsilon(t-s)}\right)\right] \\
\leq & A^{k_{n}} \exp \left(k_{n}\left\|\Delta f_{n}\right\|_{\infty}^{2}\right) .
\end{aligned}
$$

Set

$$
D_{\varepsilon, n}:=\int_{0}^{1} \int_{0}^{1} \exp \left(\lambda_{n} \frac{\left|\sum_{j=1}^{k_{n}} f_{n}\left(B_{\varepsilon t}^{j}\right)-f_{n}\left(B_{\varepsilon s}^{j}\right)\right|^{2}}{|t-s|}\right) d t d s .
$$

Then (7.16) implies that

$$
E\left[D_{\varepsilon, n}^{\frac{1}{\varepsilon}}\right] \leq A^{k_{n}} \exp \left(k_{n}\left\|\Delta f_{n}\right\|_{\infty}^{2}\right)
$$

Set $p(u):=u^{\frac{1}{2}}$. Then by Garsia's Lemma (cf. [BY82]),

$$
\begin{aligned}
\left|\sum_{j=1}^{k_{n}} f_{n}\left(B_{\varepsilon t}^{j}\right)-f_{n}\left(B_{\varepsilon s}^{j}\right)\right| & \leq \frac{8}{\sqrt{\lambda_{n}}} \int_{0}^{|t-s|}\left(\log \left(\frac{D_{\varepsilon, n}}{u^{2}}\right)\right)^{\frac{1}{2}} d p(u) \\
& \leq \frac{8 c_{\delta}}{\sqrt{\lambda_{n}}}\left(\sqrt{\log \left(D_{\varepsilon, n}\right)}+1\right)|t-s|^{\frac{1}{2}-\delta}
\end{aligned}
$$


It follows that

$$
\begin{aligned}
I I_{n}^{\varepsilon} & =P_{\gamma_{0}}\left(\left|\sum_{j=1}^{k_{n}} f_{n}\left(B_{\varepsilon t}^{j}\right)-f_{n}\left(B_{\varepsilon s}^{j}\right)\right|>L_{n}|t-s|^{\frac{1}{2}-\delta} \text { for some } 0 \leq s \leq t \leq 1\right) \\
& \leq P_{\gamma_{0}}\left(\frac{8 c_{\delta}}{\sqrt{\lambda_{n}}}\left(\sqrt{\log \left(D_{\varepsilon, n}\right)}+1\right)>L_{n}\right) \\
& =P_{\gamma_{0}}\left\{D_{\varepsilon, n}^{\frac{1}{\varepsilon}}>\exp \left(\frac{\left(\frac{L_{n} \sqrt{\lambda_{n}}}{8 c_{\delta}}-1\right)^{2}}{\varepsilon}\right)\right\} \\
& \leq \exp \left(-\frac{\left(\frac{L_{n} \sqrt{\lambda_{n}}}{8 c_{\delta}}-1\right)^{2}}{\varepsilon}\right) E\left[D_{\varepsilon, n}^{\frac{1}{\varepsilon}}\right] \\
& \leq \exp \left(-\frac{\left(\frac{L_{n} \sqrt{\lambda_{n}}}{8 c_{\delta}}-1\right)^{2}}{\varepsilon}\right) A^{k_{n}} \exp \left(k_{n}\left\|\Delta f_{n}\right\|_{\infty}^{2}\right) \\
(7.19) & =\exp \left(-\frac{\left(\frac{L_{n} \sqrt{\lambda_{n}}}{8 c_{\delta}}-1\right)^{2}}{\varepsilon}+k_{n} \log (A)+k_{n}\left\|\Delta f_{n}\right\|_{\infty}^{2}\right) \\
(7.20) & \leq \exp \left(-\frac{n+L}{\varepsilon}\right),
\end{aligned}
$$

where we used the definition of $L_{n}$ in the last step.

Combining (7.11), (7.15) and (7.20) gives

$$
\begin{aligned}
P_{\gamma_{0}}^{\varepsilon}\left(K_{L}^{c}\right) & \leq\left(\hat{c}_{1}+1\right) \sum_{n=1}^{\infty} \exp \left(-\frac{n+L}{\varepsilon}\right) \\
& \leq\left(\hat{c}_{1}+1\right) \exp \left(-\frac{L}{\varepsilon}\right) \sum_{n=1}^{\infty} \exp (-n) .
\end{aligned}
$$

Consequently,

$$
\lim _{\varepsilon \rightarrow 0} \varepsilon \log P_{\gamma_{0}}^{\varepsilon}\left(K_{L}^{c}\right) \leq-L .
$$

and the proof is complete.

\section{§8. Interacting Case}

In this section, we will prove a sample path large deviation principle for the interacting random particles introduced in Section 2. Recall that $\mathcal{M}_{\psi}:=$ $\left\{\Omega, X_{t}, \mathcal{F}, \mathcal{F}_{t}, Q_{\gamma}, \gamma \in \Gamma_{M}\right\}$ denotes the diffusion associated with the following Dirichlet form:

$$
\begin{aligned}
\mathcal{E}_{\psi}(u, v) & =\frac{1}{2} \int_{\Gamma_{M}}\langle\nabla u, \nabla v\rangle_{\gamma} \psi^{2}(\gamma) \pi(d \gamma), \\
D\left(\mathcal{E}_{\psi}\right) & =\overline{\mathcal{D}}^{\mathcal{E}_{\psi, 1}}
\end{aligned}
$$


where $\mathcal{D}$ is given by

$$
\mathcal{D}:=\left\{u \in D(\mathcal{E}) \mid \int\left(\Gamma(u, u)(\gamma)+u^{2}(\gamma)\right) \psi^{2}(\gamma) \pi(d \gamma)<\infty\right\} .
$$

The following theorem is a special case of the general result obtained in [Ebe96].

Theorem 8.1. The diffusion $\left(\Omega,\left(X_{t}\right)_{t \geq 0}, \mathcal{F}, \mathcal{F}_{t}, Q_{\gamma}, \gamma \in \Gamma_{M}\right)$ is given by

$$
d Q_{\gamma}\left|\mathcal{F}_{t}=\exp \left\{M_{t}^{\log \psi}-\frac{1}{2}\left\langle M^{\log \psi}\right\rangle_{t}\right\} d P_{\gamma}\right|_{\mathcal{F}_{t}}
$$

where $M^{\log \psi}$ stands for the martingale part of the Fukushima's decomposition of the additive functional $\log \psi\left(X_{t}\right)-\log \psi\left(X_{0}\right)$ (see [Fuk80]) and

$$
\left\langle M^{\log \psi}\right\rangle_{t}=\int_{0}^{t} \frac{\Gamma(\psi, \psi)}{\psi^{2}}\left(X_{s}\right) d s
$$

is the bracket, where $\Gamma(\psi, \psi)$ is defined as in Section 2.

Theorem 8.2. Assume $\int_{\Gamma_{M}} \exp \left(\delta \frac{\Gamma(\psi, \psi)}{\psi^{2}}(\gamma)\right) \pi(d \gamma)<\infty$ for some $\delta>$ 0 . Then there exists a subset $F_{M} \subset \Gamma_{\infty}$ with $\pi\left(F_{M}\right)=1$ such that for $\gamma_{0} \in F_{M}$ the following holds

(i) for any closed subset $C \subset C_{\gamma_{0}}\left([0,1] \rightarrow \Gamma_{M}\right)$,

$$
\limsup _{\varepsilon \rightarrow 0} \varepsilon \log Q_{\gamma_{0}}\left(X_{\varepsilon}, \in C\right) \leq-\inf _{\omega \in C} I(\omega),
$$

(ii) for any open subset $O \subset C_{\gamma_{0}}\left([0,1] \rightarrow \Gamma_{M}\right)$,

$$
\liminf _{\varepsilon \rightarrow 0} \varepsilon \log Q_{\gamma_{0}}\left(X_{\varepsilon} \in O\right) \geq-\inf _{\omega \in O} I(\omega) .
$$

In particular, if $\frac{\Gamma(\psi, \psi)}{\psi^{2}}$ is bounded, $F_{M}$ can be chosen to be equal to $\Gamma_{\infty}$.

Proof. Let $P_{\pi}(\cdot):=\int_{\Gamma_{M}} P_{\gamma}(\cdot) \pi(d \gamma)$. Denote by $E_{\pi}$ the expectation w.r.t. $P_{\pi}$. By Jensen's inequality, for any $\varepsilon>0$

$$
\begin{aligned}
E_{\pi}\left[\exp \left(\delta \int_{0}^{1} \frac{\Gamma(\psi, \psi)}{\psi^{2}}\left(X_{\varepsilon s}\right) d s\right)\right] & \leq \int_{0}^{1} E_{\pi}\left[\exp \left(\delta \frac{\Gamma(\psi, \psi)}{\psi^{2}}\left(X_{\varepsilon s}\right)\right)\right] d s \\
& =\int_{\Gamma_{M}} \exp \left(\delta \frac{\Gamma(\psi, \psi)}{\psi^{2}}(\gamma)\right) \pi(d \gamma)<\infty
\end{aligned}
$$


This implies that

$$
F_{1}:=\left\{\gamma \mid E_{\gamma}\left[\exp \left(\delta \int_{0}^{1} \frac{\Gamma(\psi, \psi)}{\psi^{2}}\left(X_{\frac{s}{k}}\right) d s\right)\right]<\infty \text { for all } k \geq 1\right\}
$$

has full $\pi$ measure. Set $F_{M}:=\Gamma_{\infty} \cap F_{1}$ and fix $\gamma_{0} \in F_{M}$. Let us prove (ii) first. Assume $O$ is open and set

$$
Z_{t}:=\exp \left\{M_{t}^{\log \psi}-\frac{1}{2}\left\langle M^{\log \psi}\right\rangle_{t}\right\}
$$

We may assume $\lambda:=\inf _{\omega \in O} I(\omega)<\infty$. For any $\delta_{1}>0$, it follows that

$$
\begin{aligned}
Q_{\gamma_{0}}\left(X_{\varepsilon}\right. & \in O) \\
& =P_{\gamma_{0}}\left(Z_{\varepsilon} ; X_{\varepsilon} \in O\right) \\
& \geq \exp \left(-\frac{\delta_{1}}{\varepsilon}\right) P_{\gamma_{0}}\left(Z_{\varepsilon}>\exp \left(-\frac{\delta_{1}}{\varepsilon}\right), X_{\varepsilon} \in O\right) \\
& \geq \exp \left(-\frac{\delta_{1}}{\varepsilon}\right)\left[P_{\gamma_{0}}\left(X_{\varepsilon} \in O\right)-P_{\gamma_{0}}\left(Z_{\varepsilon} \leq \exp \left(-\frac{\delta_{1}}{\varepsilon}\right)\right)\right]
\end{aligned}
$$

where $P_{\gamma_{0}}\left(Z_{\varepsilon} \leq \exp \left(-\frac{\delta_{1}}{\varepsilon}\right)\right)$ can be estimated as follows: for $a>0$,

$$
\begin{aligned}
P_{\gamma_{0}}\left(Z_{\varepsilon} \leq\right. & \left.\exp \left(-\frac{\delta_{1}}{\varepsilon}\right)\right) \\
= & P_{\gamma_{0}}\left(Z_{\varepsilon}^{-1} \geq \exp \left(\frac{\delta_{1}}{\varepsilon}\right)\right) \\
= & P_{\gamma_{0}}\left[\exp \left(-a M_{\varepsilon}^{\log \psi}+\frac{a}{2} \int_{0}^{\varepsilon} \frac{\Gamma(\psi, \psi)}{\psi^{2}}\left(X_{s}\right) d s\right) \geq \exp \left(\frac{a \delta_{1}}{\varepsilon}\right)\right] \\
\leq & \exp \left(-\frac{a \delta_{1}}{\varepsilon}\right) E_{\gamma_{0}}\left[\exp \left(-2 a M_{\varepsilon}^{\log \psi}-2 a^{2} \int_{0}^{\varepsilon} \frac{\Gamma(\psi, \psi)}{\psi^{2}}\left(X_{s}\right) d s\right)\right]^{\frac{1}{2}} \\
& \times E_{\gamma_{0}}\left[\exp \left(\left(2 a^{2}+a\right) \int_{0}^{\varepsilon} \frac{\Gamma(\psi, \psi)}{\psi^{2}}\left(X_{s}\right) d s\right)\right]^{\frac{1}{2}} \\
\leq & \exp \left(-\frac{a \delta_{1}}{\varepsilon}\right) D_{a}(\varepsilon),
\end{aligned}
$$

where

$$
D_{a}(\varepsilon):=E_{\gamma_{0}}\left[\exp \left(\left(2 a^{2}+a\right) \int_{0}^{\varepsilon} \frac{\Gamma(\psi, \psi)}{\psi^{2}}\left(X_{s}\right) d s\right)\right]^{\frac{1}{2}},
$$

and we have used the fact that $\exp \left(-2 a M_{\varepsilon}^{\log \psi}-2 a^{2} \int_{0}^{\varepsilon} \frac{\Gamma(\psi, \psi)}{\psi^{2}}\left(X_{s}\right) d s\right)$ is a supermartingale. 
On the other hand, for any $\delta_{2}>0$, by Theorem 7.1 there is $\varepsilon_{1}>0$ such that if $\varepsilon \leq \varepsilon_{1}$,

$$
P_{\gamma_{0}}\left(X_{\varepsilon}, \in O\right) \geq \exp \left(-\frac{\lambda+\delta_{2}}{\varepsilon}\right) .
$$

Choose $a=\frac{\lambda+2 \delta_{2}}{\delta_{1}}$. It follows from (8.1), (8.2) and (8.3) that if $\varepsilon \leq \varepsilon_{1}$,

$$
\begin{aligned}
Q_{\gamma_{0}}\left(X_{\varepsilon} \in O\right) & \geq \exp \left(-\frac{\delta_{1}}{\varepsilon}\right) \times\left[\exp \left(-\frac{\lambda+\delta_{2}}{\varepsilon}\right)-D_{a}(\varepsilon) \exp \left(-\frac{\lambda+2 \delta_{2}}{\varepsilon}\right)\right] \\
& =\exp \left(-\frac{\delta_{1}}{\varepsilon}\right) \exp \left(-\frac{\lambda+\delta_{2}}{\varepsilon}\right)\left[1-\exp \left(-\frac{\delta_{2}}{\varepsilon}\right) D_{a}(\varepsilon)\right]
\end{aligned}
$$

By the choice of $\gamma_{0}$, it is easy to see that

$$
\lim _{\varepsilon \rightarrow 0}\left[1-\exp \left(-\frac{\delta_{2}}{\varepsilon}\right) D_{a}(\varepsilon)\right]=1 .
$$

Hence, we obtain from (8.4), (8.5) that

$$
\liminf _{\varepsilon \rightarrow 0} \varepsilon \log Q_{\gamma_{0}}\left(X_{\varepsilon} \in O\right) \geq-\delta_{1}-\lambda-\delta_{2} .
$$

Since $\delta_{1}, \delta_{2}$ were arbitrary, (ii) follows.

Let us now prove (i). Because of the choice of $\gamma_{0}$, by similar arguments as used above one can see that $\lim _{\varepsilon \rightarrow 0} E_{\gamma_{0}}\left[Z_{\varepsilon}^{p}\right]=1$ for any $p>0$. For any $\delta_{1}>0$, $a>0$, we have

$$
\begin{aligned}
Q_{\gamma_{0}}\left(X_{\varepsilon} \in C\right) & =P_{\gamma_{0}}\left(Z_{\varepsilon} ; X_{\varepsilon} \in C\right) \\
& =P_{\gamma_{0}}\left(Z_{\varepsilon} ; X_{\varepsilon} \in C, Z_{\varepsilon} \leq e^{\frac{\delta_{1}}{\varepsilon}}\right)+P_{\gamma_{0}}\left(Z_{\varepsilon} ; X_{\varepsilon} \in C, Z_{\varepsilon}>e^{\frac{\delta_{1}}{\varepsilon}}\right) \\
& \leq P_{\gamma_{0}}\left(X_{\varepsilon} \in C\right) e^{\frac{\delta_{1}}{\varepsilon}}+E_{\gamma_{0}}\left[Z_{\varepsilon}^{a+1}\right] e^{-\frac{a \delta_{1}}{\varepsilon}} .
\end{aligned}
$$

It follows that

$$
\begin{aligned}
& \limsup _{\varepsilon \rightarrow 0} \varepsilon \log Q_{\gamma_{0}}\left(X_{\varepsilon} \in C\right) \\
& \quad \leq\left(\limsup _{\varepsilon \rightarrow 0} \varepsilon \log P_{\gamma_{0}}\left(X_{\varepsilon} \in C\right)+\delta_{1}\right) \vee\left(\limsup _{\varepsilon \rightarrow 0} \varepsilon \log E_{\gamma_{0}}\left[Z_{\varepsilon}^{a+1}\right]-a \delta_{1}\right) \\
& \quad \leq\left(-\inf _{\omega \in C} I(\omega)+\delta_{1}\right) \vee\left(-a \delta_{1}\right) .
\end{aligned}
$$

First letting $a \rightarrow \infty$ and then $\delta_{1} \rightarrow 0$, we prove (i). 


\section{Acknowledgement}

We thank F. Z. Gong for very useful discussions. The support of British EPSRC (grant no. GR/R91144/01), the BiBoS-Research Centre, and the DFGForschergruppe "Spectral Analysis, Asymptotic Distributions and Stochastic Dynamics" is gratefully acknowledged.

\section{References}

[AKR96a] Albeverio, S., Kondratiev, Y. G. and Röckner, M., Canonical Dirichlet operator and distorted Brownian motion on Poisson spaces, C. R. Acad. Sci. Paris, Sér. I, 323 (1996), 1179-1184.

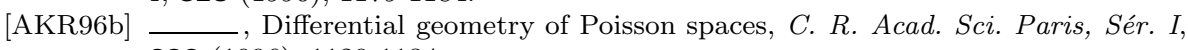
323 (1996), 1129-1134.

[AKR98a] , Analysis and geometry on configuration spaces, J. Funct. Anal., 154 (1998), 444-500.

[AKR98b] Analysis and geometry on configuration spaces. The Gibbsian case, $J$. Funct. Anal., 157 (1998), 242-291.

[AZ02] Aida, S. and Zhang, T.-S., On the small time asymptotics of diffusion processes on path groups, Pot. Anal., 16 (2002), 67-78.

[Aze80] Azencott, R., Grandes deviations et applications, Lect. Notes Math., 774, Springer, 1980.

[BY82] Barlow, M. T. and Yor, M., Semi-martingale inequalities via the GarsiaRodemich-Rumsey lemma and application to local times, J. Funct. Anal., 49 (1982), 198-229.

[Dav89] Davies, E. B., Heat kernels and spectral theory, Cambridge University Press, Cambridge, 1989.

[Doo53] Doob, J. L., Stochastic processes, Wiley and Sons, New York, London, 1953.

[DZ92] Dembo, A. and Zeitouni, O., Large deviations techniques and applications, Jones and Bartlett Publishers, Boston, London, 1992.

[Ebe96] Eberle, A., Girsanov-type transformations of local Dirichlet forms: an analytic approach, Osaka J. Math., 33 (1996), 497-531.

[Fan94] Fang, S., On the ornstein-uhlenbeck process, Stochastics Stochastics Rep., 46 (1994), 141-159.

[Fuk80] Fukushima, M., Dirichlet forms and symmetric markov processes, North-Holland, Amsterdam, 1980.

[FZ99] Fang, S. and Zhang, T.-S., On the small time behavior of ornstein-uhlenbeck processes with unbounded linear drifts, Prob. Th. Rel. Fields, 114 (1999), 487504.

[GW00] Gong, F.-Z. and Wang, F.-Y. Heat kernel estimates with application to compactness of manifolds, Preprint Bielefeld (2000).

[Hin02] Hino, M., On short time asymptotic behaviour of some symmetric diffusions on general state spaces, Pot. Anal., 16 (2002), 249-264.

[Kal97] Kallenberg, O., Foundations of modern probability, Springer, New York, London, 1997.

[KLR03] Kondratiev, Y. G., Lytvynov, E. and Röckner, M., The heat semigroup on configuration spaces, Publ. RIMS, Kyoto Univ., 39 (2003), 1-48.

[MR92] Ma, Z.-M. and Röckner, M., Introduction to the theory of (non-symmetric) Dirichlet forms, Springer, Berlin, 1992.

[MR00] _ Construction of diffusions on configuration spaces, Osaka J. Math., 37 (2000), 273-314. 
[Nor97] Norris, J. R., Heat kernel asymptotics and distance function in lipschitz riemannian manifold, Acta Math., 179 (1997), 79-103.

[Ram01] Ramirez, J. A., Short time asymptotics in dirichlet spaces, Comm. Pure Appl. Math., 54 (2001), 259-293.

[RS99] Röckner, M. and Schied, A., Rademacher's theorem on configuration spaces and applications, J. Funct. Anal., 169 (1999), 325-356.

[Sch96] Schied, A., Sample path large deviations for super-brownian motion, Prob. Th. Rel. Fields, 104 (1996), 319-347.

[Stu92] Sturm, K. T., Heat kernel bounds on manifolds, Math. Ann., 292 (1992), 149-162.

[Var67] Varadhan, S. R. S., Diffusion processes in small time intervals, Comm. Pure Appl. Math., 20 (1967), 659-685.

[Zha00] Zhang, T.-S., On the small time asymptotics of diffusion processes on hilbert spaces, Ann. Prob., 28 (2000), 537-557.

[Zha01] _ On the small time large deviations of diffusion processes on configuration spaces, Stoch. Proc. Appl., 91 (2001), 239-254. 